\title{
A Quantum Logic of Down Below
}

\author{
P. D. Bruza And D. Widdows and John Woods
}

\section{Introduction}

The logic that was purpose-built to accommodate the hoped-for reduction of arithmetic gave to language a dominant and pivotal place. Flowing from the founding efforts of Frege, Peirce, and Whitehead and Russell, this was a logic that incorporated proof theory into syntax, and in so doing made of grammar a senior partner in the logicistic enterprise. The seniority was reinforced by soundness and completeness metatheorems, and, in time, Quine would quip that the "grammar [of logic] is linguistics on purpose" [Quine, 1970, p. 15] and that "logic chases truth up the tree of grammar" [Quine, 1970, p. 35]. Nor was the centrality of syntax lost with the Gödel incompleteness results, which, except for the arithmeticization of syntax, would have been impossible to achieve.

Logic's preoccupation with language is no recent thing. In Aristotle's logic of the syllogism, the target properties of necessitation and syllogistic entailment are properties of sentences or sets of sentences of Greek. Only with the likes of Peirce and Frege is the rejection of natural language explicit, each calling for a logic whose properties would attach to elements of artificial languages, and - after Tarski - to such elements in semantic relation to non-linguistic set theoretic structures.

It is hardly surprising that mathematical logic should have given such emphasis to language, given that the motivating project of logic was to facilitate the reduction of arithmetic to an obviously analytic discipline. Still, it is also worthy of note that the historic role of logic was to lay bare the logical structure of human reasoning. Aristotle is clear on this point. The logic of syllogisms would serve as the theoretical core of a wholly general theory of real-life, two-party argumentation. Even here, the centrality of language could not be ignored. For one thing, it was obvious that real-life argumentation is transacted in speech. For a second, it was widely held (and still is) that reasoning is just soliloquial argumentation (just as argumentation is held to be reasoning in public - out loud, so to speak). Given these purported equivalences, reasoning too was thought of as linguistic. 
It is convenient to date the birth of modern mathematical logic from the appearance in 1879 of Frege's great book on the language of logic, Begriffsschrift. It is easy to think of logic as having a relatively unfettered and richly progressive course ever since, one in which even brutal setbacks could be celebrated as triumphs of metalogic. There is, however, much of intervening importance from 1904 onwards, what with developments in intuitionist, modal, many-valued and relevant logics, which in retrospect may seem to presage crucial developments in the second half of that century. Suffice it here to mention Hintikka's seminal work on epistemic logic [Hintikka, 1962], which is notable in two important respects. One is the introduction of agents as load-bearing objects of the logic. The other is the influence that agents are allowed to have on what the theory is prepared to count as its logical truths. The logical truths of this system include its indefensible sentences, where these in turn include sentences which it would be self-defeating for an agent to utter (e.g., "I can't speak a single word of English"). It is easy to see that Hintikka here allows for a sentence to be a truth of logic if its negation is pragmatically inconsistent. To this extent, the presence of agents in his logic occasions the pragmaticization of its semantics.

Agents now enter logic with a certain brisk frequency. They are either expressly there or are looming forces in theories of belief dynamics and situation semantics, in theories of default and non-monotonic reasoning, and in the incipient stirrings of logics of practical reasoning. Notable as these developments are, they all lie comfortably within the embrace of the linguistic presumption. Agents may come or go in logic, but whether here or there, they are, in all that makes them of interest to logicians, manipulators of language. What is more, notwithstanding the presence of agents, these were logics that took an interest in human reasoning rather than human reasoners. This made for a fateful asymmetry in which what human reasoners are like (or should be) is read off from what human reasoning is like (or should be).

It may be said, of course, that this is exactly the wrong way around, that what reasoning is (or should be) can only be read off from what reasoners are (and can be). Such a view one finds, for example in [Gabbay and Woods, 2001] and [Gabbay and Woods, 2003b], among logicians, and, also in the social scientific literature [Simon, 1957, Stanovich, 1999, Gigerenzer and Selten, 2001b]. Here the leading idea of the "new logic" is twofold. First, that logic's original mission as a theory of human reasoning should be re-affirmed. Second, that a theory of human reasoning must take empirical account of what human reasoners are like - what they are interested in and what they are capable of.

It is easy to see that the human agent is a cognitive being, that human 
beings have a drive to know. They desire to know what to believe and what to do. And since, whatever else it is, reasoning is an aid to cognition, a theory of human reasoning must take into account how beings like us operate as cognitive systems. Here, too, the empirical record is indispensable. It is the first point of contact between logic and cognition. In this way symbolic inference becomes "married" to computations through state (dimensional) spaces motivated from cognition which may open the door the large-scale operational symbolic inference systems. The logicians Barwise and Seligman have advocated such a marriage between logic and cognition [Barwise and Seligman, 1997, p.234]. This bears in an important way on what we have been calling the linguistic presumption. For if the empirical record is anything to go on, much of the human cognitive project is sublinguistic, and inaccessible to introspection. This, the cognition of "down below", carries consequences for the new logic. If logic is to attend to the cognizing agent, it must take the cognizer as he comes, warts and all. Accordingly, a theory of human reasoning must subsume a logic of down below.

These days, the logic of down below appears to have a certain memetic status. It is an idea whose time has come. In addition to the work of Gabbay and Woods, the idea is independently in play in a number of recent writings. In [Churchland, 1989, Churchland, 1995] we find a connectionist approach to subconscious abductive processes (cf. [Burton, 1999]). In a series of papers, Horgan and Tiensen develop a rules without representation (RWR) framework for cognitive modeling [Horgan and Tienson, 1988, Horgan and Tienson, 1989, Horgan and Tienson, 1990, Horgan and Tienson, 1992, Horgan and Tienson, 1996, Horgan and Tienson, 1999b, Horgan and Tienson, 1999a] (Cf. [Guarini, 2001]). Other non-representational orientations include [Wheeler, 2001, Sterelny, 1990, Brooks, 1991, Globus, 1992, Shannon, 1993, Thelen and Smith, 1993, Wheeler, 1994, Webb, 1994, Beer, 1995] (Cf. [Wimsatt, 1986] and [Clark, 1997]). A neural symbolic learning systemic framework is developed in [d'Avila Garcez et al., 2002, d'Avila Garcez and Lamb, 2004] and extended to abductive environments in [Gabbay and Woods, 2005, ch. 6]. Bruza and his colleagues advance a semantic space framework [Bruza et al., 2004, Bruza and Cole, 2005b, Bruza et al., 2006].

The present chapter is offered as a contribution to the logic of down below. In the section to follow, we attempt to demonstrate that the nature of human agency necessitates that there actually be such a logic. The ensuing sections develop the suggestion that cognition down below has a structure strikingly similar to the physical structure of quantum states. In its general form, this is not an idea that originates with the present authors. It is known that there exist mathematical models from the cognitive science of cognition down below that have certain formal similarities to quantum 
mechanics. We want to take this idea seriously. We will propose that the subspaces of von Neumann-Birkhoff lattices are too crisp for modelling requisite cognitive aspects in relation to subsymbolic logic. Instead, we adopt an approach which relies on projections into nonorthogonal density states. The projection operator is motivated from cues which probe human memory.

\section{Agency}

In this section our task is to orient the logic of down below by giving an overview of salient features of individual cognitive agency. Investigations of non-monotonic reasoning (NMR) have successfully provided an impressive symbolic account of human practical reasoning over the last two and half decades. The symbolic characterization of practical reasoning, however, is only part of the picture. Gärdenfors [Gärdenfors, 2000, p. 127] argues that one must go under the symbolic level of cognition. In this vein, he states, "... information about an object may be of two kinds: propositional and conceptual. When the new information is propositional, one learns new facts about the object, for example, that $x$ is a penguin. When the new information is conceptual, one categorizes the object in a new way, for example, $x$ is seen as a penguin instead of as just a bird". Gärdenfors' mention of "conceptual" refers to the conceptual level of a three level model of cognition [Gärdenfors, 2000]. How information is represented varies greatly across the different levels. The sub-conceptual level is the lowest level within which information is carried by a connectionist representation. Within the uppermost level information is represented symbolically. It is the intermediate, conceptual level, or conceptual space, which is of particular relevance to this account. Here properties and concepts have a geometric representation in a dimensional space. For example, the property of "redness" is represented as a convex region in a tri-dimensional space determined by the dimensions hue, chromaticity and brightness. The point left dangling for the moment is that representation at the conceptual level is rich in associations, both explicit and implicit. We speculate that the dynamics of associations are primordial stimuli for practical inferences drawn at the symbolic level of cognition. For example, it seems that associations and analogies generated within conceptual space play an important role in hypothesis generation. Gärdenfors ([Gärdenfors, 2000], p48) alludes to this point when he states, "most of scientific theorizing takes place within the conceptual level."

Gärdenfors' conjecture receives strong endorsement from an account of practical reasoning developed in [Gabbay and Woods, 2003a, Gabbay and Woods, 2005], in which reasoning on the ground is understood to function under economic constraints. In this essay, our own point of departure is that subsymbolic 
reasoning is valuable to human agents precisely for the economies it achieves. It will help to place this assumption in its proper context by giving a brief overview of our approach to cognitive agency.

\section{A Hierarchy of Agency Types}

It is useful to repeat the point that since reasoning is an aid to cognition, a logic, when conceived of as a theory of reasoning, must take this cognitive orientation deeply into account. Accordingly, we will say that a cognitive system is a triple of a cognitive agent, cognitive resources, and cognitive target performed in real time. (See here [Norman, 1993, Hutchins, 1995].) Correspondingly, a logic of a cognitive system is a principled description of conditions under which agents deploy resources in order to perform cognitive tasks. Such is a practical logic when the agent it describes is a practical agent.

A practical logic is but an instance of a more general conception of logic. The more general notion is reasoning that is target-motivated and resourcedependent. Correspondingly, a logic that deals with such reasoning is a Resource-Target Logic ( $R T$-logic). In our use of the term, a practical logic is a $R T$-logic relativized to practical agents.

How agents perform is constrained in three crucial ways: in what they are disposed towards doing or have it in mind to do (i.e., their agendas); in what they are capable of doing (i.e., their competence); and in the means they have for converting competence into performance (i.e., their resources). Loosely speaking, agendas here are programmes of action, exemplified by belief-revision and belief-update, decision-making and various kinds of casemaking and criticism transacted by argument. ${ }^{1}$

Agency-type is set by two complementary factors. One is the degree of command of resources an agent needs to advance or close his (or its) agendas. For cognitive agendas, three types of resources are especially important. They are (1) information, (2) time, and (3) computational capacity. The other factor is the height of the cognitive bar that the agent has set for himself. Seen this way, agency-types form a hierarchy $H$ partially ordered by the relation $C$ of commanding-greater-resources-in-support-of-higher-goalsthan. $H$ is a poset (a partially ordered set) fixed by the ordered pair $\langle C, X\rangle$ of the relation $C$ on the unordered set of agents $X$.

Human agency divides roughly into the individual and the institutional. By comparison, individual agency ranks low in $H$. For large classes of cases, individuals perform their cognitive tasks on the basis of less information and less time than they might otherwise like to have, and under limitations on the processing and manipulating of complexity. Even so, paucity must not

\footnotetext{
${ }^{1}$ Agendas are discussed at greater length in [Gabbay and Woods, 2002].
} 
be confused with scarcity. There are lots of cases in which an individual's resources are adequate for the attainment of the attendant goal. In a rough and ready way, we can say that the comparative modesty of an agent's cognitive goals inoculates him against cognitive-resource scarcity. But there are exceptions, of course.

Institutional entities contrast with human agents in all these respects. A research group usually has more information to work with than any individual, and more time at its disposal; and if the team has access to the appropriate computer networks, more fire-power than most individuals even with good PCs. The same is true, only more so, for agents placed higher in the hierarchy - for corporate actors such as NASA, and collective endeavours such as particle physics since 1970. Similarly, the cognitive agendas that are typical of institutional agents are by and large stricter than the run-ofthe-mill goals that motivate individual agents. In most things, NASA aims at stable levels of scientific confirmation, but, for individuals the defeasibly plausible often suffices for local circumstances.

These are vital differences. Agencies of higher rank can afford to give maximization more of a shot. They can wait long enough to make a try for total information, and they can run the calculations that close their agendas both powerfully and precisely. Individual agents stand conspicuously apart. He must do his business with the information at hand, and, much of the time, sooner rather than later. Making do in a timely way with what he knows now is not just the only chance of achieving whatever degree of cognitive success is open to him as regards the agenda at hand; it may also be what is needed in order to avert unwelcome disutilities, or even death. Given the comparative humbleness of his place in $H$, the human individual is frequently faced with the need to practise cognitive economies. This is certainly so when either the loftiness of his goal or the supply of drawable resources create a cognitive strain. In such cases, he must turn scantness to advantage. That is, he must (1) deal with his resource-limits and in so doing (2) must do his best not to kill himself. There is a tension in this dyad. The paucities with which the individual is chronically faced are often the natural enemy of getting things right, of producing accurate and justified answers to the questions posed by his agenda. And yet, not only do human beings contrive to get most of what they do right enough not to be killed by it, they also in varying degrees prosper and flourish. This being so, we postulate for the individual agent slight-resource adjustment strategies (SRAS), which he uses to advantage in dealing with the cognitive limitations that inhere in the paucities presently in view. We make this assumption in the spirit of Simon [1957] and an ensuing literature in psychology and economics. At the heart of this approach is the well-evidenced fact that, for ranges of cases, 
"fast and frugal" is almost as good as full optimization, and at much lower cost [Gigerenzer and Selten, 2001a]. We shall not take time here to detail the various conditions under which individuals extract outcome economies from resource limitations and target modesty, but the examples to follow will give some idea of how these strategies work.

Although resource-paucity should not be equated with resource-scarcity, it remains the case that in some sense practical agents operate at a cognitive disadvantage. It is advisable not to make too much of this. What should be emphasized is that in relation to the cognitive standards that an institutional agent might be expected to meet, the resources available to a practical agent will typically not enable him (or it) to achieve that standard. Whether this constitutes an unqualified disadvantage depends on the nature of the task the individual has set for himself and the cognitive resources available to him. For a practical agent to suffer an unqualified disadvantage, two factors must intersect in the appropriate way: his resources must be inadequate for the standard he should hit, in relation to a goal that has reasonably been set for him. So, the measure of an agent's cognitive achievement is a function of three factors: his cognitive goal; the standard required (or sufficient) for achieving that goal; and the cognitive wherewithal on which he can draw to meet that standard.

In discharging his cognitive agendas, the practical agent tends to set goals that he can attain and to be stocked with the wherewithal that makes attainment possible (and frequent). In the matter of both goals set and the execution of standards for meeting them, the individual is a satisficer rather than an optimizer. There are exceptions, of course; a working mathematician won't have a solution of Fermat's Last Theorem unless he has a full-coverage proof that is sound (and, as it happens, extremely long).

The tendency to satisfice rather than maximize (or optimize) is not what is distinctive of practical agency. This is a point to emphasize. In most of what they set out to do and end up achieving, institutional agents exhibit this same favoritism. What matters - and sets them apart from the likes of us - is not that they routinely optimize but that they satisfice against loftier goals and tougher standards.

\section{Slight-resource Adjustment Strategies}

Slight-resource adjustment strategies lie at the crux of the economy of effort, as Rescher calls it [Rescher, 1996, p.10]. They instantiate a principle of least effort, and they bear on our tendency to minimize the expenditure of cognitive assets. ${ }^{2}$ We note here some examples.

Examples are easy to come by in discussing cognitive and economic be-

\footnotetext{
${ }^{2}$ See here the classic work of George Zipf. [Zipf, 1949]
} 
haviour. More formal examples are also easy to come by, in every field of study. Statistical studies such as opinion polls always give results to within a given level of confidence (e.g., "these predictions are valid to within $\pm 3 \%$ with $95 \%$ confidence"), and part of the science of statistics lies in making reliable statements of this nature given the size of sample taken. Medical tests are often only correct to a known precision, and given the fequency of false-positives, the result of a positive test-result is often a further round of more reliable but more invasive tests.

It may be tempting to presume that such knowledge-constrained strategies are mainly confined to empirical or practical sciences, but this is far from the case. For example, mathematics is full of rules-of-thumb and famous theorems that reduce difficult problems to easy ones. These begin for many early students with the familiar division rules, such as "if a number ends in a 2 or a 5 , it is divisible by 2 or 5 ", or the more complex "if the alternating sum of the digits of a number is divisible by 11 , the number itself is divisible by 11". Such results do not produce the quotient of the division, but they may tell the student whether such a computation is worth the trouble if the goal is to end up with a whole number. More advanced division properties are embodied in results such as Fermat's Little Theorem, which states that if $p$ is prime and $1 \leq a \leq p$, then $a^{p-1} \cong 1(\bmod p)$. Like many important theorems, this only gives necessary but not sufficient conditions for a statement (in this case, the statement " $p$ is prime") to be true. However, if this necessary condition holds for enough values of $a$, we may conclude that $p$ is probably prime, which is in fact a strong enough guarantee for some efficient encryption algorithms. Even in mathematics, often regarded as the most exact and uncompromising discipline, short-cuts that are close enough are not only important, they are actively sought after.

\subsection{Hasty Generalization}

Individual cognitive agents are hasty generalizers, otherwise known as thinslicers. Hasty generalization is a $S R A S$. In standard approaches to fallacy theory and theories of statistical inference, hasty generalization is a blooper; it is a serious sampling error. This is the correct assessment if the agent's objective is to find a sample that is guaranteed to raise the conditional probability of the generalization, and to do so in ways that comport with the theorems of the applied mathematics of chance. Such is an admirable goal for agents who have the time and know-how to construct or find samples that underwrite such guarantees. But as J.S. Mill shrewdly observed, human individuals often lack the wherewithal for constructing these inferences. The business of sample-to-generalization induction often exceeds the resources of individuals and is better left to institutions. (See [Woods, 2004].) A 
related issue, even supposing that the requisitely high inductive standards are meetable in a given situation in which a practical agent finds himself, is whether it is necessary or desirable for him (or it) to meet that standard. Again, it depends on what the associated cognitive goal is. If, for example, an individual's goal is to have a reasonable belief about the leggedness of ocelots is, rather than to achieve the highest available degree of scientific certainty about it, it would suffice for him to visit the ocelot at the local zoo, and generalize hastily "Well, I see that ocelots are four-legged".

\subsection{Generic Inference}

Often part of what is involved in a human reasoner's facility with the oneoff generalization is his tendency to eschew generalizations in the form of universally quantified conditional propositions. When he generalizes hastily the individual agent is often making a generic inference. In contrast to universally quantified conditional propositions, a generic claim is a claim about what is characteristically the case. "For all $x$, if $x$ is a ocelot, then $x$ is four-legged" is one thing; "Ocelots are four-legged" is quite another thing [Krifka et al., 1995]. The first is felled by any true negative instance, and thus is brittle. The second can withstand multiples of true negative instances, and thus is elastic. There are significant economies in this. A true generic claim can have up to lots of true negative instances. So it is true that ocelots are four-legged, even though there are up to lots of ocelots that aren't four-legged. The economy of the set-up is evident: With generic claims, it is unnecessary to pay for every exception. One can be wrong in particular without being wrong in general.

Generic claims are a more affordable form of generalization than the universally quantified conditional. This is part of what explains their dominance in the generalizations that individual agents tend actually to make (and to get right, or some near thing). It must not be thought, however, that what constitutes the rightness (or some near thing) of an individual's hasty generalizations is that when he generalizes thus he generalizes to a generic claim. Although part of the story, the greater part of the rightness of those hasty generalizations arises from the fact that, in making them, an individual typically has neither set himself, nor met, the standard of inductive strength. This, together with our earlier remarks about validity, is telling. Given the cognitive goals typically set by practical agents, validity and inductive strength are typically not appropriate (or possible) standards for their attainment. This, rather than computational costs, is the deep reason that practical agents do not in the main execute systems of deductive or inductive logic as classically conceived. 


\subsection{Natural Kinds}

Our adeptness with generic inference and hasty generalization is connected to our ability to recognize natural kinds [Krifka et al., 1995, pp.63-95]. Natural kinds have been the object of much metaphysical skepticism of late [Quine, 1969], but it is a distinction that appeals to various empirical theorists. The basic idea is evident in concepts such as frame [Minsky, 1975], prototype [Smith and Medin, 1981], script [Schank and Abelson, 1977] and exemplar [Rosch, 1978]. It is possible, of course, that such are not a matter of metaphysical unity but rather of perceptual and conceptual organization.

It goes without saying that even when the goal is comparatively modest - say, what might plausibly be believed about something at hand - not every hasty generalization that could be made comes anywhere close to hitting even that target. The (defeasible) rule of thumb is this: The hasty generalizations that succeed with these more modest goals are by and large those we actually draw in actual cognitive practice. We conjecture that the comparative success of such generalizations is that they generalize to generic propositions, in which the process is facilitated by the agent's adeptness in recognizing natural kinds. In section 5, we discuss the extent to which a quantum logical framework provides a more useful model for adapting to natural kinds than either Boolean set theory or taxonomy.

\subsection{Consciousness}

A further important respect in which individual agency stands apart from institutional agency is that human agents are conscious. (The consciousness of institutions, such as it may be figuratively speaking, supervenes on the consciousness of the individual agents who constitute them.) Consciousness is both a resource and a limitation. Consciousness has a narrow bandwidth. This makes most of the information that is active in a human system at a time consciously unprocessible at that time. In what the mediaevals called the sensorium (the collective of the five senses operating concurrently), there exist something in excess of 10 million bits of information per second; but fewer than 40 bits filter into consciousness at those times. Linguistic agency involves even greater informational entropy. Conversation has a bandwidth of about 16 bits per second. ${ }^{3}$

\footnotetext{
${ }^{3}$ [Zimmermann, 1989]. Here is John Gray on the same point: "If we do not act in the way we think we do, the reason is partly to do with the bandwidth of consciousness its ability to transmit information measured in terms of bits per second. This is much too narrow to be able to register the information we routinely receive and act on. As organisms active in the world, we process perhaps 14 million bits of information per second. The bandwidth of consciousness is around eighteen bits. This means that we have conscious access to about a millionth of the information we daily use to survive" [Gray, 2002, p. 66].
} 
The narrow bandwidth of consciousness bears on the need for cognitive economy. It helps elucidate what the scarcity of information consists in. We see it explained that at any given time the human agent has only slight information by the fact that if it is consciously held information there is a bandwidth constraint which regulates its quantity. There are also devices that regulate consciously processible information as to type. A case in point is informational relevance. When H.P. Grice issued the injunction, "Be relevant", he left it undiscussed whether such an imperative could in fact be honoured or ignored by a conscious act of will. There is evidence that the answer to this question is "No"; that, in lot's of cases, the mechanisms that steer us relevantly in the transaction of our cognitive tasks, especially those that enable us to discount or evade irrelevance, are automatic and pre-linguistic [Gabbay and Woods, 2003a]. If there is marginal capacity in us to heed Grice's maxim by consciously sorting out relevant from irrelevant information, it is likely that these informational relevancies are less conducive to the closing of cognitive agendas than the relevancies that operate "down below". Thus vitally relevant information often can't be processed consciously, and much of what can is not especially vital. ${ }^{4}$

Consciousness can claim the distinction of being one of the toughest problems, and correspondingly, one of the most contentious issues in the cognitive sciences. Since the agency-approach to logic subsumes psychological factors, it is an issue to which the present authors fall heir, like it or not. Many researchers accept the idea that information carries negative entropy, that it tends to impose order on chaos. ${ }^{5}$ If true, this makes consciousness a thermodynamically expensive state to be in, since consciousness is a radical suppressor of information. Against this are critics who abjure so latitudinarian a conception of information [Hamlyn, 1990] and who remind us that talk about entropy is most assured scientifically for closed systems (and that ordinary individual agents are hardly that).

The grudge against promiscuous "informationalism", in which even physics goes digital [Wolfram, 1984], is that it fails to explain the distinction between energy-to-energy transductions and energy-to-information transformations [Tallis, 1999, p. 94]. Also targeted for criticism is the view that

\footnotetext{
${ }^{4}$ Consider here taxonomies of vision in which implicit perception has a well-established place [Rensink, 2000].

${ }^{5}$ Thus Colin Cherry: "In a descriptive sense, entropy is often referred to as a 'measure of disorder' and the Second Law of Thermodynamics as stating that 'systems can only proceed to a state of increased disorder; as time passes, entropy can never decrease.' The properties of a gas can change only in such a way that our knowledge of the positions and energies of the particles lessens; randomness always increases. In a similar descriptive way, information is contrasted, as bringing order out of chaos. Information, then is said to be 'like' negative energy" [Cherry, 1966, p. 215].
} 
consciousness arises from or inheres in neural processes. If so, "[h]ow does the energy impinging on the nervous system become transformed into consciousness?" [Tallis, 1999, p. 94].

In the interests of economy, we decline to join the metaphysical fray over consciousness. The remarks we have made about consciousness are intended not as advancing the metaphysical project but rather as helping characterize the economic limitations under which individual cognitive agents are required to perform.

Consciousness is tied to a family of cognitively significant issues. This is reflected in the less than perfect concurrence among the following pairs of contrasts:

1. conscious $\mathrm{v}$ unconscious processing

2. controlled $\mathrm{v}$ automatic processing

3. attentive $\mathrm{v}$ inattentive processing

4. voluntary $\mathrm{v}$ involuntary processing

5. linguistic $\mathrm{v}$ nonlinguistic processing

6. semantic $\mathrm{v}$ nonsemantic processing

7. surface $\mathrm{v}$ depth processing

What is striking about this septet of contrasts is not that they admit of large intersections on each side, but rather that their concurrence is approximate at best. For one thing, "tasks are never wholly automatic or attentive, and are always accomplished by mixtures of automatic and attentive processes" [Shiffrin, 1997, p. 50]. For another, "depth of processing does not provide a promising vehicle for distinguishing consciousness from unconsciousness (just as depth of processing should not be used as a criterial attribute for distinguishing automatic processes ..." [Shiffrin, 1997, p. 58]). Indeed "[s]ometimes parallel processing produces an advantage for automatic processing, but not always .... Thoughts high in consciousness often seem serial, probably because they are associated with language, but at other times consciousness seems parallel ..." [Shiffrin, 1997, p. 62].

It is characteristic of agents of all types to adjust their cognitive targets upwards as the cognitive resources for attaining them are acquired. A practical agent may take on commitments previously reserved for agents of higher rank if, for example, he is given the time afforded by a tenured position in a university, the information stored in the university's library and in his own PC, and the fire-power of his university's mainframe. In like fashion, institutional agents constantly seek to expand their cognitive resources (while driving down the costs of their acquisition, storage and deployment), so that even more demanding targets might realistically be set. 
Accordingly, agents tend toward the enhancement of cognitive assets when this makes possible the realization of cognitive goals previously unattainable (or unaffordable). Asset enhancement is always tied to rising levels of cognitive ambition. In relation to cognitive tasks adequately performed with present resources, an interest in asset enhancement is obsessive beyond the range of what would count as natural and proportionate improvements upon what is already adequately dealt with.

\subsection{Subsymbolic reasoning}

Practical reasoning is reasoning performed by practical agents, and is therefore subject to economic constraints. In this connection, we advance the following conjecture: It may well be that because such associations are formed below the symbolic level of cognition, significant cognitive economy results. This is not only interesting from a cognitive point of view, but also opens the door to providing a computationally tractable practical reasoning systems, for example, operational abduction to drive scientific discovery in biomedical literature [Bruza et al., 2004, Bruza et al., 2006]

The appeal of Gärdenfors' cognitive model is that it allows inference to be considered not only at the symbolic level, but also at the conceptual (geometric) level. Inference at the symbolic level is typically a linear, deductive process. Within a conceptual space, inference takes on a decidedly associational character because associations are often based on similarity (e.g., semantic or analogical similarity), and notions of similarity are naturally expressed within a dimensional space. For example, Gärdenfors states that a more natural interpretation of "defaults" is to view them as "relations between concepts". ${ }^{6}$ This is a view which flows into the account which follows: the strength of associations between concepts change dynamically under the influence of context. This, in turn, influences the defaults haboured within the symbolic level of cognition.

It is important to note the paucity of representation at the symbolic level and reflect how symbolic reasoning systems are hamstrung as a result. In this connection, Gärdenfors ([Gärdenfors, 2000, p. 127]) states, “..information about categorization can be quite naturally transfered to propositional information: categorizing $x$ as an emu, for example, can be expressed by the proposition " $x$ is an emu". This transformation into the propositional form, however, tends to suppress the internal structure of concepts. Once one formalizes categorizations of objects by predicates in a first-order language, there is a strong tendency to view the predicates as primitive, atomic notions and to forget that there are rich relations among concepts that dis-

\footnotetext{
${ }^{6}$ In the theory of Gabbay and Woods, default reasoning is a core slight-resource compensation strategy.
} 
appear when put into standard logical formalism."

The above contrast between the conceptual and symbolic levels raises the question as to what are the implications for providing an account of practical reasoning. Gärdenfors states that concepts generate "expectations that result in different forms of non-monotonic reasoning", which are summarized as follows:

\section{Change from a general category to a subordinate}

When shifting from a basic category, e.g., "bird" to a subordinate category, e.g., "penguin", certain default associations are given up (e.g., "Tweety flies"), and new default properties may arise (e.g., "Tweety lives in Antarctica").

\section{Context effects}

The context of a concept triggers different associations that "lead to nonmonotonic inferences". For example, Reagan has default associations "Reagan is a president", "Reagan is a republican" etc., but Reagan seen in the context of Iran triggers associations of "Reagan" with "arms scandal", etc.

\section{The effect of contrast classes}

Properties can be relative, for example, "a tall Chihuahua is not a tall dog" ([Gärdenfors, 2000, p. 119]),. In the first contrast class "tall" is applied to Chihuahuas and the second instance it is applied to dogs in general. Contrast classes generate conceptual subspaces, for example, skin colours form a subspace of the space generated by colours in general. Embedding into a subspace produces non-monotonic effects. For example, from the fact that $x$ is a white wine and also an object, one cannot conclude that $x$ is a white object (as it is yellow).

\section{Concept combination}

Combining concepts results in non-monotonic effects. For example, metaphors ([Gärdenfors, 2000, p. 130]), Knowing that something is a lion usually leads to inferences of the form that it is alive, that it has fur, and so forth. In the combination, stone lion, however, the only aspect of the object that is lionlike is its shape. One cannot conclude that a stone lion has the other usual properties of a lion, and thus we see the non-monotonicity of the combined concept.

An example of the non-monotonic effects of concept combination not involving metaphor is the following: A guppy is not a typical pet, nor is guppy is a typical fish, but a guppy is a typical pet fish.

In short, concept combination leads to conceptual change. These correspond to revisions of the concept and parallel belief revisions modelled at 
the symbolic level, the latter having received thorough examination in the artificial intelligence literature.

The preceding characterization of the dynamics of concepts and associated non-monotonic effects is intended to leave the impression that a lot of what happens in connection with practical reasoning takes place within a conceptual (geometric) space, or a space of down-below. What is more, this impression may provide a foothold towards realizing genuine operational systems. This would require that at least three issues be addressed. The first is that a computational variant of the conceptual level of cognition is necessary. Secondly, the non-monotonic effects surrounding concepts would need to be formalized and implemented. Thirdly, the connection between these effects and NMR at the symbolic level needs to be specified. This account will cover aspects related to the first two of these questions. Computational approximations of conceptual space will be furnished by semantic space models which are emerging from the fields of cognition and computational linguistics. Semantic space models not only provide a cognitively motivated basis to underpin human practical reasoning, but from a mathematical perspective, they show a marked similarity with quantum mechanics (QM) [Aerts and Czachor, 2004]. This introduces the tantalizing and unavoidably speculative prospect of formalizing aspects of human practical reasoning via QM.

\section{Semantic space: computational approximations of conceptual space}

To illustrate how the gap between cognitive knowledge representation and actual computational representations may be bridged, the Hyperspace Analogue to Language (HAL) semantic space model is employed [Lund and Burgess, 1996, Burgess et al., 1998]. HAL produces representations of words in a high dimensional space that seem to correlate with the equivalent human representations. For example, “...simulations using HAL accounted for a variety of semantic and associative word priming effects that can be found in the literature...and shed light on the nature of the word relations found in human word-association norm data"[Lund and Burgess, 1996]. Given an $n$-word vocabulary, HAL computes an $n \times n$ matrix constructed by moving a window of length $l$ over the corpus by one word increment ignoring punctuation, sentence and paragraph boundaries. All words within the window are considered as co-occurring with the last word in the window with a strength inversely proportional to the distance between the words. Each row $i$ in the matrix represents accumulated weighted associations of word $i$ with respect to other words which preceded $i$ in a context window. Conversely, column $i$ represents accumulated weighted associations with words that appeared 


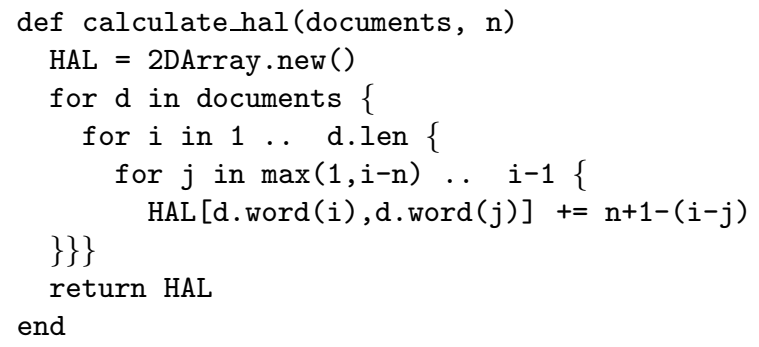

Figure 1.1. Algorithm to compute the HAL matrix for a collection of documents. It is assumed that the documents have been pruned of stop words and punctuation.

after $i$ in a window. For example, consider the text "President Reagan ignorant of the arms scandal", with $l=5$, the resulting HAL matrix $H$ would be:

\begin{tabular}{|c|c|c|c|c|c|c|c|}
\hline & arms & ig & of & pres & reag & scand & the \\
\hline arms & 0 & 3 & 4 & 1 & 2 & 0 & 5 \\
\hline ig & 0 & 0 & 0 & 4 & 5 & 0 & 0 \\
\hline of & 0 & 5 & 0 & 3 & 4 & 0 & 0 \\
\hline pres & 0 & 0 & 0 & 0 & 0 & 0 & 0 \\
\hline reag & 0 & 0 & 0 & 5 & 0 & 0 & 0 \\
\hline scand & 5 & 2 & 3 & 0 & 1 & 0 & 4 \\
\hline the & 0 & 4 & 5 & 2 & 3 & 0 & 0 \\
\hline
\end{tabular}

Table 1.1. A simple semantic space computed by HAL

If word precedence information is considered unimportant the matrix $S=H+H^{T}$ denotes a symmetric matrix in which $S[i, j]$ reflects the strength of association of word $i$ seen in the context of word $j$, irrespective of whether word $i$ appeared before or after word $j$ in the context window. The column vector $S_{j}$ represents the strengths of association between $j$ and other words seen in the context of the sliding window: the higher the weight of a word, the more it has lexically co-occurred with $j$ in the same context(s). For example, table 1.2 illustrates the vector representation for "Reagan" taken from a matrix $S$ computed from a corpus of 21578 Reuters ${ }^{7}$ news feeds

\footnotetext{
${ }^{7}$ The Reuters-21578 collection is standard test collection used for research into automatic text classification
} 
president (5259), administration (2859), trade (1451), house (1426), budget (1023), congress (991), bill (889), tax (795), veto (786), white (779), japan (767), senate (726), iran (687), billion (666), dlrs (615), japanese (597), officials (554), arms (547), tariffs (536) ..

Table 1.2. Example representation of the word "Reagan"

taken from the year 1988. (The weights in the table are not normalized). Highly weighted associations reflect Reagan in his presidential role dealing with congress, tax, vetoes etc. In addition, the more highly weighted association reflect a default-like character, e.g., "president" and "administration". Associations with lower weights seem to reflect the trade war with Japan ("japan", "tariffs") and the Iran-contra scandal ("Iran", "arms"). In other words, the representation of Reagan represents a mixture of different "senses" of Reagan. This facet is intuitively similar to the QM phenomenon of a particle being in a state of superposition.

HAL is an exemplar of a growing ensemble of computational models emerging from cognitive science, which are generally referred to as semantic spaces [Lund and Burgess, 1996, Burgess et al., 1998, Lowe, 2000, Lowe, 2001, Landauer and Dumais, 1997, Landauer et al., 1998, Patel et al., 1997, Schütze, 1998, Levy and Bullinaria, 1999, Sahlgren, 2002]. Even though there is ongoing debate about specific details of the respective models, they all feature a remarkable level of compatibility with a variety of human information processing tasks such as word association. Semantic spaces provide a geometric, rather than propositional, representation of knowledge. They can be considered to be approximations of conceptual space proposed by Gärdenfors [Gärdenfors, 2000], and of reasoning down below as proposed by [Gabbay and Woods, 2003a, Gabbay and Woods, 2005].

Within a conceptual space, knowledge has a dimensional structure. For example, the property colour can be represented in terms of three dimensions: hue, chromaticity, and brightness. Gärdenfors argues that a property is represented as a convex region in a geometric space. In terms of the example, the property "red" is a convex region within the tri-dimensional space made up of hue, chromaticity and brightness. The property "blue" would occupy a different region of this space. A domain is a set of integral dimensions in the sense that a value in one dimension(s) determines or affects the value in another dimension(s). For example, the three dimensions defining the colour space are integral since the brightness of a colour will affect both its saturation (chromaticity) and hue. Gärdenfors extends the notion of properties into concepts, which are based on domains. The 
concept "apple" may have domains taste, shape, colour, etc. Context is modelled as a weighting function on the domains, for example, when eating an apple, the taste domain will be prominent, but when playing with it, the shape domain will be heavily weighted (i.e., it's roundness). One of the goals of this article is to provide both a formal and operational account of this weighting function.

Observe the distinction between representations at the symbolic and conceptual levels. At the symbolic level "apple" can be represented as the atomic proposition apple $(x)$. However, within a conceptual space (conceptual level), it has a representation involving multiple inter-related dimensions and domains. Colloquially speaking, the token "apple" (symbolic level) is the tip of an iceberg with a rich underlying representation at the conceptual level. Gärdenfors points out that the symbolic and conceptual representations of information are not in conflict with each other, but are to be seen as "different perspectives on how information is described".

Barwise and Seligman [Barwise and Seligman, 1997] also propose a geometric foundation to their account of inferential information content via the use of real-valued state spaces. In a state space, the colour "red" would be represented as a point in a tri-dimensional real-valued space. For example, brightness can be modelled as a real-value between white (0) and black (1). Integral dimensions are modelled by so called observation functions defining how the value(s) in dimension(s) determine the value in another dimension. Observe that this is a similar proposal, albeit more primitive, to that of Gärdenfors as the representations correspond to points rather than regions in the space.

Semantic space models are also an approximation of Barwise and Seligman state spaces whereby the dimensions of the space correspond to words. A word $j$ is a point in the space. This point represents the "state" in the context of the associated text collection from which the semantic space was computed. If the collection changes, the state of the word may also change. Semantic space models, however, do not make provision for integral dimensions. An important intuition for the following is the state of a word in semantic space is tied very much with its "meaning", and this meaning is context-sensitive. Further, context-sensitivity will be realized by state changes of a word.

In short, HAL, and more generally semantic spaces, are a promising, pragmatic means for knowledge representation based on text. They are computational approximations, albeit rather primitively, of Gärdenfors' conceptual space. Moreover, due to their cognitive track record, semantic spaces would seem to be a fitting foundation for considering realizing computational variants of human reasoning. Finally, it has been shown that a semantic space 
is formally a density matrix, a notion from QM [Aerts and Czachor, 2004, Bruza and Cole, 2005a]. This opens the door to exploring further connections with QM.

\section{Bridging Semantic Space and Quantum Mechanics}

HAL exemplifies how a semantic space model assigns each word in a given vocabulary a point in a finite dimensional vector space. Lowe [Lowe, 2001] formalizes semantic space models as a quadruple $\langle A, B, F, M\rangle$ where

- $B$ is a set of $m$ basis elements

- $A$ is a function which maps the co-occurrence frequencies between words in a vocabulary $V$ and the basis elements so each $w \in V$ is represented by a vector $\left(A\left(b_{1}, w\right), \ldots, A\left(b_{m}, w\right)\right)$

- $F$ is a function which maps pairs of vectors onto continuous valued quantity. The interpretation of $F$ is often "semantic similarity" between the two vectors in question.

- $M$ is a transformation which takes one semantic space and maps it into another, for example via dimensional reduction

A semantic space ${ }^{8} S$ is an instance of the range of the function $A$. That is, $S$ is a $m \times n$ matrix where the columns $\{1, \ldots, n\}$ correspond to vector representations of words.A typical method for deriving the vocabulary $V$ is to tokenize the corpus from which the semantic space is computed and remove non information bearing words such as "the", "a", etc. The letters $u, v, w$ will be used to identify individual words.

The interpretation of the basis elements corresponding to the rows $\{1 \ldots m\}$ depends of the type of semantic space in question. For example, table 3 illustrates that HAL produces a square matrix in which the rows are also interpreted as representations of terms from the vocabulary $V$. In contrast, a row in the semantic space models produced by Latent Semantic Analysis [Landauer et al., 1998] corresponds to a text item, for example, a whole document, a paragraph, or even a fixed window of text, as above. The value $S[t, w]=x$ denotes the salience $x$ of word $w$ in text $t$. Informationtheoretic approaches are sometimes use to compute salience. Alternatively, the (normalized) frequency of word $w$ in context $t$ can be used.

For reasons of a more straightforward embedding of semantic space into $\mathrm{QM}$, we will focus on square, symmetric semantic spaces $(m=n)$. The following draws from [van Rijsbergen, 2004]

\footnotetext{
${ }^{8}$ Bear in mind that the term "space" should not be interpreted as a "vector space". This unfortunate blurring between "matrix" and "space" in the technical sense occurs because "semantic space" is a term from the cognitive science literature.
} 
A word $w$ is represented as a column vector in $S$ :

$$
|w\rangle=\left(\begin{array}{c}
w_{1} \\
\vdots \\
w_{n}
\end{array}\right)
$$

The notation on the LHS is called a ket, and originates from quantum physicist Paul Dirac. Conversely, a row vector $v=\left(v_{1}, \ldots, v_{n}\right)$ is denoted by the bra $\langle v|$.

Multiplying a ket by a scalar $\alpha$ is as would be expected:

$$
\alpha|w\rangle=\left(\begin{array}{c}
\alpha w_{1} \\
\vdots \\
\alpha w_{n}
\end{array}\right)
$$

Addition of vectors $|u\rangle+|v\rangle$ is also as one would expect. In Dirac notation, the scalar product of two $n$-dimensional real ${ }^{9}$ valued vectors $u$ and $v$ produces a real number:

$$
\langle u \mid v\rangle=\sum_{i=1}^{n} u_{i} v_{i}
$$

The outer product $|u\rangle\langle u|$ produces a $n \times n$ symmetric matrix. Vectors $u$ and $v$ are orthogonal iff $\langle u \mid v\rangle=0$. Scalar product allows the length of a vector to be defined: $\|u\|=\sqrt{\langle u \mid u\rangle}$. A vector $|u\rangle$ can be normalized to unit length $(\|u\|=1)$ by dividing each of its components by the vector's length: $\frac{1}{\|u\|}|u\rangle$.

A Hilbert space is a complete ${ }^{10}$ inner product space. In the formalization to be presented in ensuing sections, a semantic space $S$ is an $n$-dimensional real-valued Hilbert space using Euclidean scalar product as the inner product.

A Hilbert space allows the state of a quantum system to be represented. It is important to note that a Hilbert space is an abstract state space meaning QM does not prescribe the state space of specific systems such as electrons. This is the responsibility of a physical theory such as quantum electrodynamics. Accordingly, it is the responsibility of semantic space theory to offer the specifics: In a nutshell, a ket $|w\rangle$ describes the state of "meaning"

\footnotetext{
${ }^{9} \mathrm{QM}$ is founded on complex vector spaces. We restrict our attention to finite vector spaces of real numbers.

${ }^{10}$ The notion of a "complete" vector space should not be confused with "completeness" in logic. The definition of a completeness in a vector space is rather technical, the details of which are not relevant to this account.
} 
of a word $w$. It is akin to a particle in QM. The state of a word changes due to context effects in a process somewhat akin to quantum collapse. This in turn bears on practical inferences drawn due to context effects of word seen together with other words as described above.

In QM, the state can represent a superposition of potentialities. By way of illustration consider the state $\sigma$ of a quantum bit, or qubit as:

$$
|\sigma\rangle=\alpha|0\rangle+\beta|1\rangle
$$

where $\alpha^{2}+\beta^{2}=1$. The vectors $|0\rangle$ and $|1\rangle$ represent the characteristic states, or eigenstates, of "off" and "on". Eigenstates are sometimes referred to as pure, or basis states. They can be pictured as defining orthogonal axes in a 2- D plane:

$$
\alpha|0\rangle=\left(\begin{array}{l}
0 \\
1
\end{array}\right)
$$

and

$$
\alpha|1\rangle=\left(\begin{array}{l}
1 \\
0
\end{array}\right)
$$

The state $\sigma$ is a linear combination of eigenstates. Hard though it is to conceptualize, the linear combination allows the state of the qubit to be a mixture of the eigenstates of being "off" and "on" at the same time.

In summary, a quantum state encodes the probabilities of its measurable properties, or eigenstates. The probability of observing the qubit being off (i.e., $|0\rangle$ is $\alpha^{2}$ ). Similarly, $\beta^{2}$ is the probability of observing it being "on".

The above detour into QM raises questions in relation to semantic space. What does it mean that a word is a superposition - a "mixture of potentialities"? What are the eigenstates of a word?

\subsection{Mixed and eigenstates of a word meaning}

Consider the following traces of text from the Reuters-21578 collection:

- President Reagan was ignorant about much of the Iran arms scandal

- Reagan says U.S to offer missile treaty

- Reagan seeks more aid for Central America

- Kemp urges Reagan to oppose stock tax. 
Each of these is a window which HAL will process accumulating weighted word associations in relation to the word "Reagan". In other words, included in the HAL vector for "Reagan" are associations dealing with the Iran-contra scandal, missile treaty negotiations with the Soviets, stock tax etc. The point is when HAL runs over the full collection, the vector representation for "Reagan" is a mixture of eigenstates, whereby an eigenstate corresponds to a particular "sense", or "characteristic meaning" of the concept "Reagan". For example, Reagan, in the political sense, in the sense dealing with the Iran-Contra scandal, etc. The senses of a concept are equivalent of the eigenstates of a particle in QM [Bruza and Cole, 2005a, Aerts et al., 2005, Widdows and Peters, 2003].

Consider once again the HAL matrix $H$ computed from the text "President Reagan ignorant of the arms scandal". As mentioned before, $S=$ $H+H^{T}$ is a real symmetric matrix. Consider a set of $y$ text windows of length $l$ which are centred around a word $w$. Associated with each such text window $j, 1 \leq j \leq m$, is a semantic space $S_{j}$. It is assumed that the semantic space is $n$-dimensional, whereby the $n$ dimensions correspond to a fixed vocabulary $V$ as above. The semantic space around word $w$, denoted by $S_{w}$, can be calculated by the sum:

$$
S_{w}=\sum_{j=1}^{y} S_{j}
$$

The above formula provides a toehold for computing a semantic space in terms of a sum of semantic spaces; each constituent semantic space corresponding to a specific sense of the concept $w$. By way of illustration, Let the concept $w$ be "Reagan" and assume there are a total of $y$ traces centred on the word "Reagan", $x$ of which deal with the Iran-contra issue. These $x$ traces can be used to construct a semantic space using equation 1.7. Let $S_{i}$ denote this semantic space. Its associated probability $p_{i}=\frac{x}{y}$. Assume the concept $w$ has $m$ senses. As each sense $i$ represents a particular state of $w$, each can be represented as a semantic space $S_{i}$ with an associated probability.

$$
S_{w}=p_{1} S_{1}+\ldots+p_{m} S_{m}
$$

where $p_{1}+\ldots+p_{m}=1$.

This formula expresses that the semantic space around a concept $w$ can be conceived of as a linear combination of semantic spaces around senses of $w$. The formula is intuitively close to an analogous formula from QM whereby a density matrix can be expressed as a probability mixture of density matrices [Barndorff-Nielsen et al., 2003, p. 778]. A density matrix 
represents the state of a quantum system. A density matrix $\rho$ represents a basis state if it has a single eigenvector (eigenstate) $|e\rangle$ whereby $\rho=|e\rangle\langle e|$. The vector $e$ is termed a state vector and is assumed to be normalized to unit length, i.e., $\langle e \mid e\rangle=1$. Otherwise a density matrix $\rho$ represents a "mixed" state (superposition). A density matrix corresponding to a mixed state can be expressed as a weighted combination of density matrices corresponding to basis states. There is no requirement that the state vectors of the pure states are orthogonal to one another. This is a very important point. Intuitively, it is unrealistic to require the senses of a concept to be orthogonal. For this reason, the term "sense" will be used to denote the basis state of a word meaning, rather than "eigenstate", because, in QM, eigenstates are assumed to be mutually orthogonal.

The connection between the notions of semantic space and density matrix have been detailed elsewhere [Aerts and Czachor, 2004, Bruza and Cole, 2005a]. As mentioned in the introduction, there are various semantic space models presented in the literature. Each will involve a different rendering as density matrix. The method adopted in this account rests on the intuition the ket $\left|e_{i}\right\rangle$ in each semantic space $S_{i}$ of equation 1.8 corresponds to a state vector representing a sense of concept $w$. A density matrix $\rho_{i}$ can be formed by the product $\left|e_{i}\right\rangle\left\langle e_{i}\right|$. Building on this, a density matrix $\rho_{w}$ corresponding to the semantic space $S_{w}$ can be constructed as follows.

$$
\rho_{w}=p_{1} \rho_{1}+\ldots+p_{m} \rho_{m}
$$

Importantly, no assumption of orthogonality has been made.

This approach to representing a semantic space in a state contrasts approaches using the spectral decomposition of the semantic space [Aerts and Czachor, 2004, Aerts and Gabora, 2005]. As the semantic space $S_{w}$ is a symmetric matrix, the spectral decomposition of SVD allows $S_{w}$ to be reconstructed, where $k \leq n$ :

$$
\begin{aligned}
S_{w} & =\sum_{i=1}^{k}\left|e_{i}\right\rangle d_{i}\left\langle e_{i}\right| \\
& =\sum_{i=1}^{k} d_{i}\left|e_{i}\right\rangle\left\langle e_{i}\right| \\
& =d_{1}\left|e_{1}\right\rangle\left\langle e_{1}\left|+\ldots+d_{k}\right| e_{k}\right\rangle\left\langle e_{k}\right|
\end{aligned}
$$

This equation parallels the one given in equation 1.9. The eigenvalues $d_{i}$ relate to the probabilities of the associated eigenvectors (eigenstates in QM terminology). Each eigenstate $\left|e_{i}\right\rangle$ contributes to the linear combination via the density matrix $\left|e_{i}\right\rangle\left\langle e_{i}\right|$. The eigenstates $\left|e_{i}\right\rangle$ of $S_{w}$ should ideally 
correspond to the senses of word $w$. Unfortunately, this does not bear out in practice. A fundamental problem is that the eigenstates $\left|e_{i}\right\rangle$ computed by SVD are orthogonal, and in reality the senses of a word $w$ need not be. (See [Bruza and Cole, 2005a] for more details).

\subsection{The collapse of meaning in the light of context}

We continue by connecting the above development of quantum mechanics in semantic space to Gärdenfors' views on the interaction of context and the meaning of concepts. He states, "The starting point is that, for some concepts, the meaning of the concept is determined by the context in which it occurs" [Gärdenfors, 2000, p.119]. Context effects manifest in relation to contrast classes. In the introduction, the Chihuahua showed how property tall is relative, "a tall Chihuahua is not a tall dog". He also illustrates how contrast classes manifest in word combinations. Consider, "red" in the following combinations, "red book", "red wine", "red hair", "red skin", "red soil". Gärdenfors argues contrast classes generate conceptual subspaces, for example, skin colours form a subspace of the space generated by colours in general. In other words, each of the combinations involving "red" results in a separate subspace representing the particular quality of "red", for example, the quality of "red" would actually be "purple" when "red" is seen in the context of "wine".

The collapse of word meaning can be thought of in terms of the quantum collapse of the particle but with an important difference: The collapse due to context may not always result in a basis state because the context may not be sufficient to fully resolve the sense in question. By way of illustration, consider "Reagan" in the context of "Iran". For the purposes of discussion, assume there are two possible senses. The first deals with the Iran-contra scandal, and the other deals with hostage crisis at the American embassy in Teheran. The distinction between a measurement due to context and a physical measurement possibly has its roots in human memory. Matrix models of human memory also contain the notion of superimposed memory states, and it has been argued, "The superposition of memory traces in a vector bundle resulting from a memory retrieval has often been considered to be a noisy signal that needs to be 'cleaned up' [i.e., full collapse onto a basis state as in QM]. The point we make here is that this is not necessarily so and that the superposition of vectors [after retrieval] is a powerful process that adds to the flexibility of memory processes." (Emphasis ours) [Wiles et al., 1994].

This distinction requires a less stringent notion of collapse as maintained within QM. Consider a concept $w$ considered in the light of some context, for example, other words. The context is denoted generically by $X$. The 
effect of context $X$ is brought about by a projection operator $P_{x}$. Assuming the density matrix $\rho_{w}$ corresponding to a concept $w$, the collapse of meaning in the light of context $X$ is characterized by the following equation:

$$
P_{x} \rho_{w}=p \rho_{w}^{x}
$$

where $p$ denotes the probability of collapse and $\rho_{w}^{x}$ is the state of $w$ after the "collapse" of its meaning.

In terms of $\mathrm{QM}, \rho_{w}$ is an "observable" meaning an observable physical quantity. An observable is represented by a self-adjoint operator. As $\rho_{w}$ is a real symmetric matrix, it is therefore also a self-adjoint operator on Hilbert space. (Recall that semantic space is a Hilbert space). This is consistent with the second axiom of QM [Byron and Fuller, 1992]. Even though this equation has the form of an eigenvalue problem, the value $p$ is not an eigenvalue. It is a theorem that the eigenstates of a self adjoint operator belonging to different eigenvalues must be orthogonal, a requirement which is too strong for word meanings as was motivated earlier. Nevertheless, it will be be shown later that $p$ derives from the geometry of the space as do eigenvalues.

The previous equation is also consistent with the third axiom of QM as the result of "measurements of the observable $\left[\rho_{w}\right]$ " is an element of "the spectrum of the operator". In our case, the spectrum is specified by the probability mixture given in equation 1.9 , but more of the flexibility of this equation is exploited than is the case in QM. The key to this flexibility revolves around the fact that the sum of density matrices is a density matrix. By way of illustration, equation 1.9 can be equivalently written as the probability mixture:

$$
\rho_{w} \quad=p_{1} \rho_{1}+p_{2} \rho_{2}
$$

where $p_{1}+p_{2}=1$. Let $\rho_{1}$ correspond to the state of "Reagan" in the context of "Iran" and $\rho_{2}$ the state of "Reagan" in all other contexts. Assume, that "Reagan" is seen in the context of "Iran". The projection operator $P_{x}$ collapses $\rho_{w}$ onto $\rho_{1}$ with probability $p_{1}$. Unlike, QM, the state $\rho_{1}$ is not a basis state but corresponds to a partially resolved sense. Let the Iran-contra sense be denoted $|c\rangle$ and the Iranian embassy hostage crisis be denoted $|h\rangle$. In the light of this example, the density matrix corresponding to the state after collapse due to "Iran" would be of the form $\rho_{1}=p_{c}|c\rangle\left\langle c\left|+p_{h}\right| h\right\rangle\langle h|$, where $p_{c}+p_{h}=1$.

It has been argued in [Bruza and Cole, 2005a] that in terms of this running example many would assume the "Iran-contra" sense of "Reagan" when "Reagan" is seen in the context of "Iran". This phenomenon may have its roots in cognitive economy. Full resolution requires processing, and to avoid 
this processing, humans "guess" the more likely sense (In the example, $p_{c}$ happens to be substantially greater than $p_{h}$ ). In other words, we cautiously put forward the conjecture that collapse of meaning and abductive processes go hand in hand to fully resolve the sense, i.e., collapse onto a basis state. Even though "full" collapse eventually results, the process is not direct as is the the case of the collapse in QM. ${ }^{11}$.

The running example reveals something of the nature of the projection operator $P_{x}$. If $P_{x}$ is orthogonal to a sense $\left|e_{i}\right\rangle$ represented by the density matrix $\rho_{i}=\left|e_{i}\right\rangle\left\langle e_{i}\right|$, then $P_{x}$ projects this sense onto the zero vector $|0\rangle$. (Note the corresponding density matrix is $|0\rangle\langle 0|$ ). If the projection $P_{x}$ is not orthogonal to a sense $\left|e_{i}\right\rangle$, then it has the effect of retrieving those senses out of the combination expressed in equation 1.9. This is not unlike the notion of a cue which probes human memory. Cues can be used to access memory in two ways; via matching or retrieval processes. Matching entails the "comparison of the test cue(s) with the information stored in memory" [Humphreys et al., 1989, p 41.]. This process measures the similarity of the cue(s) and the memory representation. The output of this process is a scalar quantity (i.e., a single numeric value representing the degree or strength of the match). Memory tasks which utilise this access procedure include recognition and familiarity tasks. Retrieval involves the "recovery of qualitative information associated with a cue" [Humphreys et al., 1989, p 141.]. This information is modelled as a vector of feature weights. Retrieval tasks include free recall, cued-recall, and indirect production tasks.

The intuition we will attempt to develop is that collapse of word meaning due to context is akin to a cued-recall retrieval operation driven by the projector $P_{x}$ on a given density matrix corresponding to the state of a word meaning. The probability of collapse $p$ is a function of the scalar quantity resulting from matching.

In the matrix model of memory [Humphreys et al., 1989], memory representations can include items, contexts or, combinations of items and contexts (associations). Items can comprise stimuli, words, or concepts. Each item is modelled as a vector of feature weights. Feature weights are used to specify the degree to which certain features form part of an item. There are two possible levels of vector representation for items. These include:

- modality specific peripheral representations (e.g., graphemic or phonemic representations of words)

- modality independent central representations (e.g., semantic represe-

\footnotetext{
${ }^{11}$ For a more detailed discussion of how the logic of abduction engages with the cognitive economy of practical agency, see [Gabbay and Woods, 2005]. For the link between abduction and semantic space, see [Bruza et al., 2006]
} 
natations of words)

In our case, our discussion will naturally focus on the latter due to assumption that semantic spaces deliver semantic representations of words. For example, the "Reagan" vector $|r\rangle$ from the semantic space $S_{r}$ illustrates a "modality independent central representation".

Context can be conceptualised as a mental representation (overall holistic picture) of the context in which items, or events have occurred. (e.g., "Reagan" in the context of "Iran"). Context is also modelled as a vector of feature weights. Following from this, context is $X$ is assumed to be represented by a ket $|x\rangle$. In the case of the running example, the "Iran" vector $|i\rangle$ drawn from the semantic space $S_{i}$ could be employed as a context vector.

Memories are associative by nature and unique representations are created by combining features of items and contexts. Several different types of associations are possible [Humphreys et al., 1989]. The association of interest here is a two way association between a word $|w\rangle$ and a context $|x\rangle$. In the matrix model of memory, an association between context and a word is represented by an outer product; $|w\rangle\langle x|$. Seeing a given word (a target) in the context of other words (cue) forms an association which probes memory. Observe with respect to the running example how the probe $|r\rangle\langle i|$ embodies both the cue of the probe "Iran" and the target "Reagan".

In the light of the above brief digression into a matrix model of human memory, one possibility is to formalize the projector $P_{x}$ as the probe $|w\rangle\langle x|$. The object being probed is a density matrix which is not a superposition of memory traces but of semantic spaces hinged around a particular word or concept. Equation 1.8 and its density matrix equivalent (equation 1.9) reflect this superposition, however in this case the traces, in their raw form, are windows of text.

In short, viewing the collapse of meaning in terms of retrieval and matching processes in memory refines the collapse equation 1.10 as follows. Let $|w\rangle$ be a target concept and $|x\rangle$ be the context. Firstly, collapse of meaning is characterized by projecting the probe into the memory corresponding to the state of the target word $w$. The collapse equates with retrieving a new state of meaning reflecting the change of meaning of $w$ in light of the context.

$$
P_{x} \rho_{w}=p \frac{|w\rangle\langle x| \rho_{w}}{f\left(\left\langle x\left|\rho_{w}\right| w\right\rangle\right)}=p \rho_{w}^{x}
$$

The probability $p$ of collapse is assumed to be a function ${ }^{12}$ of the match between the probe and the memory:

\footnotetext{
${ }^{12}$ Further research is needed to provide the specifics of this function which will take into account issues such as decay processes in memory
} 


$$
p=f\left(\left\langle x\left|\rho_{w}\right| w\right\rangle\right)
$$

Motivating the collapse of meaning by means of the matrix model of memory introduces a deviation from orthodox QM. After application of the probe $|w\rangle\langle x|$, the the state after the collapse, denoted $\rho_{w}^{x}$ is not guaranteed to be density matrix. This deviation from orthodox QM is not solely a technical issue. It may well be that there are different qualities of probe. For example, "Reagan" in the context of "Iran" would intuitively involve a projection of the global "Reagan" semantic space onto a subspace dealing with "Iran". On the other hand, consider "lion" in the context of "stone". In this case, the result after the application of the context would seem to be considerably outside the "lion" space as a "stone lion" does not share many of the attributes of a living one. It would seem, then, a projection operator is not the appropriate mechanism, but rather a more general linear operator which can project "outside" the space. In the latter case, equating $P_{x}$ with the probe $|w\rangle\langle x|$ is arguably justified as such probes in the matrix model of memory briefly described earlier are transformations of the space, rather than projections into it. An alternative view is that "stone lion" is a result of concept combination and mechanisms other than projection operators are required to suitably formalize it. For example, Aerts and Gabora [Aerts and Gabora, 2005] resort to tensor products for concept combination. These are slippery issues requiring a clean distinction between context effects and concept combination. More research is needed to clarify these issues in relation to a logic of down below.

It remains to provide a characterization of $P_{x}$ as an orthodox projector as typified by the "Reagan in the context of "Iran" example. In order to do this, the senses $B=\left\{\left|e_{1}\right\rangle, \ldots,\left|e_{m}\right\rangle\right\}$ are assumed to form a basis. (The assumption here is linear independence, which is a weaker assumption than assuming the $\left|e_{i}\right\rangle$ 's are mutually orthogonal, i.e., an orthonomal basis as is commonly seen in orthodox QM). The set $B$ represents the basis of the space $S_{w}$ in relation to $\rho_{w}$. Let $B_{x}=\left\{\left|x_{1}\right\rangle, \ldots,\left|x_{r}\right\rangle\right\}$ and $B_{y}=\left\{\left|y_{1}\right\rangle, \ldots,\left|y_{m-r}\right\rangle\right\}$ such that $B_{x} \cup B_{y}=B$. The set $B_{x}$ is the basis of the subspace $S_{x}$ due to context $X$. The complementary space is denoted $S_{y}$. By way of illustration in terms of the running example, $B_{x}=\left\{\left|x_{1}\right\rangle,\left|x_{2}\right\rangle\right\}$ would corresponds to the two senses of "Reagan" in the context of "Iran" previously introduced as $\left|e_{c}\right\rangle$ and $\left|e_{h}\right\rangle$. Though complementary spaces, $S_{x}$ and $S_{y}$ are not assumed to be orthogonal. Consequently, the projection operator $P_{x}$ is "oblique" rather than orthogonal. Once again, this is a deviation from orthodox QM, but nevertheless faithful to the underlying intuition behind projection operators. As stated earlier, the projection operator $P_{x}$ "retrieves" the relevant senses out of the probability mixture (equation 1.9), that is $P_{x}\left|x_{i}\right\rangle=\left|x_{i}\right\rangle$, for $x_{i} \in\left\{\left|x_{1}\right\rangle, \ldots,\left|x_{r}\right\rangle\right\}$. These are the so called fixed points of the projector 
$P_{x}$. As a consequence, the density matrix form of the fixed points also holds as $P_{x}\left(\left|x_{i}\right\rangle\left\langle x_{i}\right|\right)=\left(P_{x}\left|x_{i}\right\rangle\right)\left\langle x_{i}|=| x_{i}\right\rangle\left\langle x_{i}\right|$. This establishes that $P_{x}$ will retrieve the density matrix form of the relevant senses expressed in equation 1.9 .

$B_{n \times m}$ is an $n \times m$ matrix with columns

$$
\left.\left[\left|x_{1}\right\rangle\left|x_{2}\right\rangle \cdots\left|x_{r}\right\rangle|| y_{1}\right\rangle\left|y_{2}\right\rangle \cdots\left|y_{m-r}\right\rangle\right]=\left[X_{n \times r} \mid Y_{n \times(m-r)}\right]
$$

The projection operator $P_{x}$ retrieves those fixed points relevant to the context. All other senses are projected onto the zero vector $|0\rangle$ :

$$
\begin{aligned}
P_{x} B & =P_{x}[X \mid Y] \\
& =\left[P_{x} X \mid P_{x} Y\right] \\
& =[X \mid 0]
\end{aligned}
$$

For the case $m=n$, the matrix $B$ has an inverse $B^{-1}$ so the makeup of the required projection operator is given by:

$$
P_{x}=[X \mid 0] B^{-1}=B\left(\begin{array}{cc}
I_{r} & 0 \\
0 & 0
\end{array}\right) B^{-1}
$$

With an eye on operational deployment on a large scale, a simple algorithmic construction of $P_{x}$ is based on the intuition that those senses which are not orthogonal to the cue should be retrieved from the linear combination of $m$ senses (equation 1.9):

$$
B_{x}=\left\{\left|e_{i}\right\rangle \mid\left\langle x\left|\rho_{i}\right| x\right\rangle>0,1 \leq i \leq m\right\}
$$

(Recall that $\left.\rho_{i}=\left|e_{i}\right\rangle\left\langle e_{i}\right|\right)$. In terms of the running example, $B_{x}=\left\{\left|e_{c}\right\rangle,\left|e_{h}\right\rangle\right.$, the two senses relevant to "Reagan" seen in the context of "Iran".

The scalar $\left\langle x\left|\rho_{i}\right| x\right\rangle$ decomposes as follows:

$$
\begin{aligned}
\left\langle x\left|\rho_{i}\right| x\right\rangle & =\left\langle x\left|\left(\left|e_{i}\right\rangle\left\langle e_{i}\right|\right)\right| x\right\rangle \\
& =\left(\left\langle x \mid e_{i}\right\rangle\right)^{2} \\
& =\cos ^{2} \theta_{i} \\
& =a_{i}
\end{aligned}
$$

where $\cos \theta_{i}$ is the angle between $|x\rangle$ and $\left|e_{i}\right\rangle$. In the second last line the equivalence between Euclidean scalar product and cosine was employed due to the vectors being normalized to unit length. This value reflects how much the given sense is being activated to the level $a_{i}$ by the cue $|x\rangle$. Stated otherwise, $a_{i}$ reflects the strength with which the sense $\rho_{i}$ is aligned with the cue $|x\rangle$. All senses $\left|e_{i}\right\rangle$ in the basis $B_{x}$ will have a positive activation value $a_{i}$. By appropriately scaling the values $a_{i}$, the effect of projector $P_{x}$ can now be expressed as a probability mixture: 


$$
P_{x} \rho_{w}=p_{1} \rho_{1}+\ldots+p_{r} \rho_{r}
$$

where $\rho_{i}=\left|e_{i}\right\rangle\left\langle e_{i}\right|$, for all $\left|e_{i}\right\rangle \in B_{x}$ and $p_{1}+\ldots+p_{r}=1$. The import of the last equation is that the effect of the projector $P_{x}$ results in a density matrix.

\subsection{The probability of collapse}

It is illustrative to examine how in the light of the running example the scalar value resulting from the matching process determines the probability of collapse (equation 1.12). First, the effect of the cue "Iran" via the context vector $|i\rangle$ is shown. The "memory" to be probed derives from the target "Reagan" and is denoted by the density matrix $\rho_{r}$.

$$
\begin{aligned}
\langle i| \rho_{r} & =\langle i|\left(p_{1} \rho_{1}+\ldots+p_{m} \rho_{m}\right) \\
& =p_{1}\langle i| \rho_{1}+\ldots+p_{m}\langle i| \rho_{m}
\end{aligned}
$$

Recall that each of the $m$ constituent density matrices $\rho_{i}$ derives from a particular sense of "Reagan" denoted $e_{i}$. Therefore the previous equation can be written as,

$$
\begin{aligned}
\langle i| \rho_{r} & =p_{1}\langle i|\left(\left|e_{1}\right\rangle\left\langle e_{1}\right|\right)+\ldots+p_{m}\langle i|\left(\left|e_{m}\right\rangle\left\langle e_{m}\right|\right) \\
& =p_{1}\left(\left\langle i \mid e_{1}\right\rangle\right)\left\langle e_{1}\right|+\ldots+p_{m}\left(\left\langle i \mid e_{m}\right\rangle\right)\left\langle e_{m}\right| \\
& =p_{1} \cos \theta_{1}\left\langle e_{1}\right|+\ldots+p_{m} \cos \theta_{m}\left\langle e_{m}\right|
\end{aligned}
$$

The salient facet of the last line is those senses that are not orthogonal to the context vectors will be retrieved $\left(\cos \theta_{i}>0\right)$ and will contribute to the probability of collapse. This accords with the intuitions expressed in the previous section. In the running example, these senses were denoted $\left|e_{c}\right\rangle$ and $\left|e_{h}\right\rangle$. So,

$$
\langle i| \rho_{r}=p_{c} \cos \theta_{c}\left\langle e_{c}\right|+p_{h} \cos \theta_{h}\left\langle e_{h}\right|
$$

A second aspect of the matching is post multiplying with the target vector "Reagan", denoted $|r\rangle$ :

$$
\begin{aligned}
\left(p_{c} \cos \theta_{c}\left\langle e_{c}\left|+p_{h} \cos \theta_{h}\left\langle e_{h}\right|\right) \mid r\right\rangle\right. & =p_{c} \cos \theta_{c}\left(\left\langle e_{c} \mid r\right\rangle\right)+p_{h} \cos \theta_{h}\left(\left\langle e_{h} \mid r\right\rangle\right) \\
& =p_{c} \cos \theta_{c} \cos \psi_{c}+p_{h} \cos \theta_{h} \cos \psi_{h} \\
& =p_{c} m_{c}+p_{h} m_{h}
\end{aligned}
$$

The angles $\cos \psi$ reflects how strongly the the sense correlates with the given target. It can be envisaged as a measure of significance of the given sense with the target $|r\rangle$. The scores due to matching of the probe with memory are reflected by the scalars $m_{c}$ and $m_{h}$. These are modified by associated probabilities of the respective senses. Finally, the two terms are added to return a single scalar. The probability of collapse is assumed to be a function of this value. 


\subsection{Summary}

The preceding development has centred around providing an account of the collapse of meaning in the light of context. It is important that the formalization rests on non-orthogonal density matrices, which is in contrast to the orthogonal approach used in the SCOP model [Aerts and Gabora, 2005]. The approach presented here draws inspiration from a cue which probe human memory and describes collapse of meaning in terms of memory cues. The notion of a "probe" is not foreign to QM. The most useful probes of the various wave functions of atoms and molecules are the various forms of spectroscopy. In spectroscopy, an atom or molecule starting with some wave function (represented by a density matrix) is probed with light, or some other particle. The light interacts with the molecule and leaves it in another state. This process is analogous to the probing of memory just described. Chemical physics also shares another similarity with our account in the sense that the underlying density matrices cannot be assumed to be orthogonal. Nonorthogonal density matrix perturbation theory has arisen to deal with nonorthogonal density matrices and may turn out to be a relevant area for formalizing additional aspects of a logic of "down below". The analogy should be mindfully employed, however. Human memory is a vast topic abundant with texture and nuance, not to mention strident debate. However we feel investigations into the memory literature can bear further fruit in relation to a QM inspired account of a logic of "down below". The matrix model of memory described above has been extended to provide an account of analogical mapping [Wiles et al., 1994]. In our opinion, it is reasonable to assume that analogical reasoning has roots in subsymbolic logic. Dunbar [Dunbar, 1999] concludes from cognitive studies that scientists frequently resort to analogies when there is not a straightforward answer to their current problem. Therefore, analogical reasoning sometimes plays a crucial role in hypothesis formation which is fundamental to abduction[Gabbay and Woods, 2005, Chapter 7]. Reasoning, then, becomes highly confounded with memory processes. Consider the "Tweety" example described earlier. When one learns that "Tweety is a penguin", it is debatable whether any reasoning takes place at all. We would argue that the example can be explained in terms of probes to memory and the associated dynamics of defaults emerge out of context effects. We have argued such probes bear a striking similarity to quantum collapse.

\section{Quantum Logic and Conceptual Generalization}

A proposal for reasoning at the subsymbolic level must give an account for how conceptual structures may arise from perceptual observations. For example, in Word and Object, Quine [Quine, 1960, p. 25] famously challenged 
philosophers to give an account for how a hearer might reliably deduce that a speaker who utters the word "gavagai" upon seeing a rabbit actually means "rabbit", instead of "part of a rabbit", or a member of some other class such as "rabbit or guppy", or even "rabbit or Reagan". In other words, how might a conceptual logic give rise to a recognition and representation of natural kinds, in such a way that this logic is cognitively beneficial?

It is known that some logics are more amenable to inductive learning than others, and that direct adherence to the Boolean distributive law effectively prevents the sort of smoothing or closure operations that may lead to the formation of natural kinds (see[Widdows and Higgins, 2004]). For example, since Boolean logic is modelled on set theory and the union of the set of rabbits and the set of frogs is a perfectly well-formed set, the concept "rabbit or guppy" is as natural as the concept of "rabbit" in Boolean logic. At the other extreme, a single-inheritance taxonomic logic (based, for example, on phylogenetic inheritance) may overgeneralize by assuming that the disjunction of "rabbit" and "guppy" must be the lowest common phylogenetic ancestor "vertebrate". This would lead also to unfortunate consequences, such as the presumption that, since a rabbit makes a good pet for a child and a guppy makes a good pet for a child, any vertebrate makes a good pet for a child.

Compared with the discrete extreme of Boolean classification, and the opposite extreme of a single-inheritance taxonomy, the vector lattice of quantum logic presents and attractive middle ground. There are distinctly well-formed concepts represented by lines and planes, there is a natural closure or smoothing operator defined by the linear span of a set, and there is a scope for multiple inheritance (since a line is contained is many different planes and an $m$-dimensional subspace is contained in many $m+n$ dimensional subspaces). Some practical evidence for the usefulness of the linear span as a disjunction of two concept vectors was provided by the experiments in [Widdows, 2003], in which the removal of a pair of concepts using negated quantum disjunction proved greatly more effective than Boolean negation at the task of removing unwanted keywords and their synonyms. The argument that projection onto subspaces of a vector space can be used as a solution to the age-old problem of learning from incomplete experience has been made one of the mainstays of Latent Semantic Analysis, by [Landauer and Dumais, 1997] and others.

It should also be noted that the use of a pure quantum logic for concept generalization in semantic space leads to problems of its own, as one would expect with any attempt to apply such a simple mathematical model to a wholesale description of language. In particular, quantum disjunctions may often overgenerate, because of the nature of the linear independence and 
the operation of taking the linear span. In practice, vectors that are very close to one another in semantic space may still be linearly independent, and will thus generate a large subspace that does not reflect that fact that the vectors were in fact drawn from a small region of this subspace. This danger is illustrated in Figure 1.2, which depicts two groups of three vectors in a 3 -dimensional vector space. In the left hand picture, the vectors $A, B$ and $C$ are orthogonal and can be used to generate the whole of the space. The vectors $D, E$ and $F$, far from being orthogonal, have high mutual similarity. However, since these vectors are still linearly independent, they can still be used to generate the whole of the space. In other words, the quantum disjunctions $A \vee B \vee C$ and $D \vee E \vee F$ are identical. This seems quite contrary to intuition, which would suggest that the concept $D \vee E \vee F$ should be much more specific than the concept $A \vee B \vee C$. A practical drawback of this overgeneration is that a search engine that used quantum disjunction too liberally would be likely to generate results that would only be judged relevant by users willing for their queries to be extrapolated considerably.

There is a natural way to fix this problem in the formalism, and it bears an interesting relation to the observation that non-orthogonal vectors and subspaces give rise to subtly related non-commuting density matrices. In the diagram, the vector $E$ lies nearly but not quite upon the line from $D$ to $F$. To simplify the description of the local situation, a reasonable approximation would be to represent $E$ by its projection onto the subspace $D \vee F$. This would amount to making the assertion " $E$ is between $D$ and $F$ ", which might not be exact, but from a human standpoint is certainly true. To generalize from this example, it would be reasonable to say that a vector $B$ can be approximately derived from a set $A_{1}, \ldots, A_{j}$ if distance between $B$ and the projection of $B$ onto $A_{1} \vee \ldots \vee A_{j}$ is small. Defining 'small' in practice is a subtle challenge, and to some extent is in the eye of the beholder - to some, Liszt is adequately represented as being somewhere between Beethoven and Brahms, but to some, his music has special qualities independently of both.

Such a discussion suggest that one of the requirements for a working quantum logic of semantic space is the ability to model automatically the "natural dimension" of a sample of points. This problem will have many variants, and different solutions will be appropriate for different users. However, there are some general techniques such as the Isomap algorithm [Tenenbaum et al., 2000] that provide dimensional decompositions of this sort, even for samples of points taken from nonlinear submanifolds of vector spaces. From a cognitive point of view, such a dimensional simplification is to be expected and indeed preferred. From microscopic observation and subsequent progress in chemistry and physics, we know that the surface of a wooden tabletop is a 

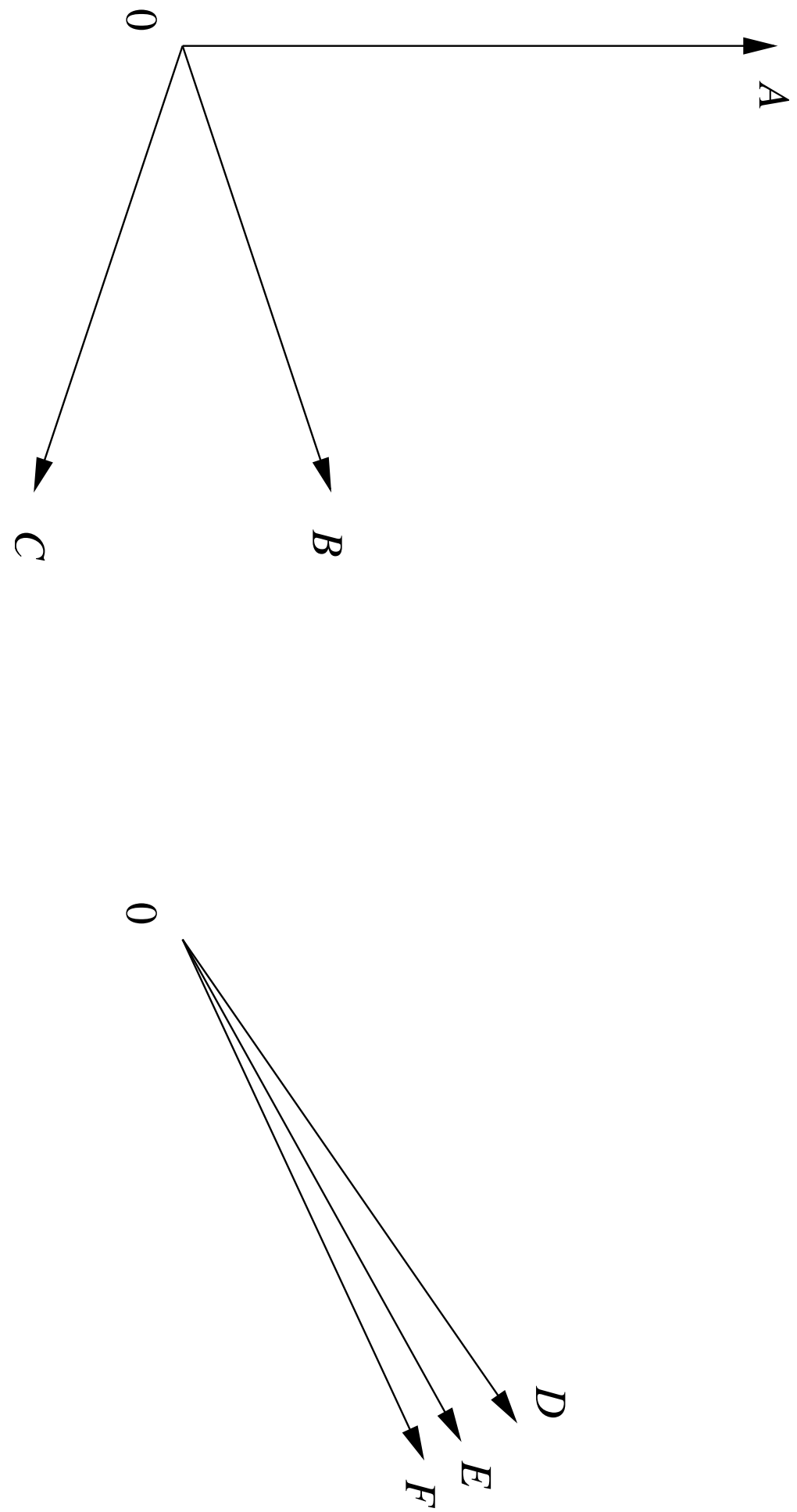

Figure 1.2. Orthogonal vectors in 3-space compared with 3 similar vectors. 
complex 3-dimensional structure, which may have a detailed fractal surface and according to some physical theories may consist of particles that need several more dimensions to be represented correctly. However, even to those humans who are well versed in such scientific precision, the tabletop is for all practical purposes a 2-dimensional structure, and you can certainly describe the whereabouts of any perceptual object on the table at a relevant scale of reference by giving two coordianates.

The challenges for adapting the vector space model to describing semantics and perception do not end here, of course. Many of the vector space axioms (such as the underlying assumption that vectors form a commutative group under addition) are seriously off the mark when viewed from a cognitive perspective. The purpose of this discussion is not to convince the reader that these problems have been completely solved, but that the immediate drawbacks of a naive implementation of quantum logic and concept formation in semantic space can be anticipated by a more careful consideration of the cognitive and logical goals of the system, whereupon plausible solutions can be found using existing mathematics.

\section{Summary and Conclusions}

A logic that is shaped by the empirical make-up of reasoning agents is subject to the same experimental challenges and limitations that affect the investigation of human subjects quite generally. The interior of the atom is, in well-known ways, difficult to access, but the interior of the reasoning agent throws up accessibility difficulties of an entirely different order. Experimental psychology, to take the most obvious example, has had to learn how to flourish despite the collapse of behaviourism and introspectivism. A great part of its success, such as it is, is owed to the skill with which it organizes its theoretical outputs around strongly plausible conjectures. In a rough and ready way, conjecturing is what one does in the absence of observation. In this regard, we are reminded of the grand conjecture with which Planck launched quantum theory itself, an idea whose immediate import in 1900 was the unification of the laws of black body radiation. In taking a quantum approach to the logic of reasoning down below, two sources of conjecture merge. In probing the down below, we conjecture in ways that incorporate the conjectures of quantum mechanics. Of course, by now there is ample empirical confirmation of quantum theory, as well as encouraging empirical support for cognitive psychology in some of its manifestations, but neither of those desirable outcomes would have been possible without the founding conjectures around which the original theories organized themselves. It is the same way with the logic of down below. A reliable empirical understanding of it has no chance of occurring spontaneously. It must be 
preceded by theoretical speculation. Eddington once quipped that theories are "put-up jobs", anticipating Quine's crack that theories are "free for the thinking up". These, of course, are jokes. The fact is that the practice of scientific conjecture is respectable to the degree that it conforms to the canons of abductive logic. One of the marks of an abductively successful conjecture is its narrative coherence with what is known observationally about the subject in question. [Thagard, 1989], [Gabbay and Woods, 2005]. Smooth narratives identify possible scenarios. This is how we find ourselves positioned here. We have sketched what we take to be a coherent narrative of the quantum character of reasoning down below. To the extent that we have succeeded in this, we have outlined a possible theory for such reasoning. What remains now is to sort out ways in which the theory might be made responsive to observational test. Initial steps in this direction have been made in the realm of text-mining and search, a field which benefits from the comparative ease of empirical measurement and hypothesis testing. Whether the theory provides a truly useful model of cognitive processes will require different observational methods.

\section{Acknowledgments}

The first author acknowledges National ICT Australia which is funded by the Australian Government's Department of Communications, Information Technology, and the Arts and the Australian Research Council through Backing Australia's Ability and the ICT Research Centre of Excellence programs. The third author thanks Dov Gabbay and Kent Peacock for helpful advice, and, for its financial support, the Engineering and Physical Sciences Research Council of the United Kingdom. Thanks to Ian Turner for helpful comments of a technical nature.

\section{BIBLIOGRAPHY}

[Aerts and Czachor, 2004] D. Aerts and M. Czachor. Quantum Aspects of Semantic Analysis and Symbolic Artificial Intelligence. Journal of Physics A-Mathematical and General, 37:L123-L132, 2004. http://uk.arxiv.org/abs/quant-ph/0309022.

[Aerts and Gabora, 2005] D. Aerts and L. Gabora. A Theory of Concepts and Their Combinations II: A Hilbert Space Representation. Kybernetes, 34:176-205, 2005. http://uk.arxiv.org/abs/quant-ph/0402205.

[Aerts et al., 2005] D. Aerts, J. Broekaert, and L. Gabora. A Case for Applying an Abstracted Quantum Formalism to Cognition. In M.H. Bickhard and R. Campbell, editors, Mind in Interaction. John Benjamins: Amsterdam, 2005.

[Barndorff-Nielsen et al., 2003] O.E. Barndorff-Nielsen, R.D. Gill, and P.E Jupp. On quantum statistical inference. Journal of the Royal Statistical Society, 65(4):775-816, 2003.

[Barwise and Seligman, 1997] J. Barwise and J. Seligman. Information flow: The logic of distributed systems. Cambridge University Press, 1997.

[Beer, 1995] R.D. Beer. Comptuational and dynamical languages for autonomous agents. In R. Port and T. van Gelder, editors, Mind as Motion: Explorations in the Dynamics of Cognition, pages 121-147. Cambridge, MA: MIT Press/Bradford Books, 1995. 
[Brooks, 1991] R.A. Brooks. Intelligence without representation. Artificial Intelligence, 47:139-159, 1991.

[Bruza and Cole, 2005a] P. Bruza and R. Cole. Quantum Logic of Semantic Space: An Exploratory Investigation of Context Effects in Practical Reasoning . In S. Artemov, H. Barringer, A. S. d'Avila Garcez, L. C. Lamb, and J. Woods, editors, We Will Show Them: Essays in Honour of Dov Gabbay, volume 1, pages 339-361. London: College Publications, 2005.

[Bruza and Cole, 2005b] P.D. Bruza and R.J. Cole. A Bare Bones Approach to Literature-Based Discovery: An analysis of the Raynaud's/fish-oil and migrainemagnesium discoveries in semantic space. In A. Hoffman, H. Motoda, and T. Scheffer, editors, Discovery Science, 8th International Conference, DS 2005, Singapore, October 8-11, 2005, Proceedings, volume 3735 of Lecture Notes in Artificial Intelligence, pages 84-98. Springer, 2005.

[Bruza et al., 2004] P.D. Bruza, D.W. Song, and R.M. McArthur. Abduction in semantic space: Towards a logic of discovery. Logic Journal of the IGPL, 12(2):97-110, 2004.

[Bruza et al., 2006] P.D. Bruza, R.J. Cole, Z. Abdul Bari, and D. Song. Towards operational abduction from a cognitive perspective. In L. Magnani, editor, Abduction and Creative Inferences in Science, Logic Journal of the IGPL. Oxford University Press, 2006.

[Burgess et al., 1998] C. Burgess, K. Livesay, and K. Lund. Explorations in context space: words, sentences, discourse. Discourse Processes, 25(2\&3):211-257, 1998.

[Burton, 1999] Robert G. Burton. A neurocomputational approach to abduction. Mind, 9:257-265, 1999.

[Byron and Fuller, 1992] F.W Byron and R.W. Fuller. The Mathematics of Classical and Quantum Physics. Dover Publications, 1992.

[Cherry, 1966] C. Cherry. On Human Communication. Cambridge, MA: MIT Press, 1966.

[Churchland, 1989] Paul Churchland. A Neurocomputational Perspective: The nature of mind and the structure of science. Cambridge, MA: MIT Press, 1989.

[Churchland, 1995] Paul M. Churchland. The Engine of Reason, The Seat of the Soul. Cambridge, MA: The MIT Press, 1995.

[Clark, 1997] A. Clark. Being There: Putting Brain, Body and World Together Again. Cambridge, MA: MIT Press/Bradford Books, 1997.

[d'Avila Garcez and Lamb, 2004] A.S. d'Avila Garcez and L.C. Lamb. Reasoning about time and knowledge in neural-symbolic learning systems. In S. Thrum and B. Schoelkopk, editors, Advances in Neural Information Processing Systems 16: Proceedings of the NIPS 2003 Conference, Vancouver, BC, 2004. Cambridge, MA: MIT Press.

[d'Avila Garcez et al., 2002] A.S. d'Avila Garcez, K. Broda, and Dov M. Gabbay. Neural-Symbolic Learning Systems: Foundations and Applications. Berlin: SpringerVerlag, 2002.

[Dunbar, 1999] K. Dunbar. How scientists build models invivo science as a window on the scientific mind. In L. Magnani, editor, Model-Based Reasoning in Scientific Discovery, pages 85-99. Kluwer Academic/Plenum Publishers, 1999.

[Gabbay and Woods, 2001] Dov M. Gabbay and John Woods. The new logic. Logic Journal of the IGPL, 9:157-190, 2001.

[Gabbay and Woods, 2002] Dov M. Gabbay and John Woods. Formal approaches to practical reasoning: A survey. In Dov M. Gabbay, Ralph H. Johnson, Hans Jürgen Ohlbach, and John Woods, editors, Handbook of the Logic of Argument and Inference: The Turn Towards the Practical, volume 1 of Studies in Logic and Practical Reasoning, pages 445-478. Amsterdam: North-Holland, 2002.

[Gabbay and Woods, 2003a] Dov M. Gabbay and John Woods. Agenda Relevance: A Study in Formal Pragmatics, volume 1 of A Practical Logic of Cognitive Systems. Amsterdam: North-Holland, 2003. 
[Gabbay and Woods, 2003b] Dov M. Gabbay and John Woods. Normative models of rationality: The disutility of some approaches. Logic Journal of IGPL, 11:597-613, 2003.

[Gabbay and Woods, 2005] Dov M. Gabbay and John Woods. The Reach of Abduction: Insight and Trial, volume 2 of A Practical Logic of Cognitive Systems. Amsterdam: North-Holland, 2005.

[Gärdenfors, 2000] P. Gärdenfors. Conceptual Spaces: The Geometry of Thought. MIT Press, 2000.

[Gigerenzer and Selten, 2001a] G. Gigerenzer and R. Selten, editors. Bounded Rationality: The Adaptive Toolbox. Cambridge, MA: MIT Press, 2001.

[Gigerenzer and Selten, 2001b] G. Gigerenzer and R. Selten. Rethinking rationality. In Bounded Rationality: The Adaptive Toolbox, pages 1-12. Cambridge, MA: MIT Press, 2001.

[Globus, 1992] G. Globus. Towards a non-computational cognitive neuroscience. Journal of Cognitive Neuroscience, 4:299-310, 1992.

[Gray, 2002] John Gray. Straw Dogs: Thoughts on Humans and Other Animals. London: Granta, 2002.

[Guarini, 2001] Marcello Guarini. A defence of connectionism against the "SYNTACTIC" argument. Synthese, 128:287-317, 2001.

[Hamlyn, 1990] D.W. Hamlyn. In and Out of the Black Box. Oxford: Basil Blackwell, 1990.

[Hintikka, 1962] Jaakko Hintikka. Knowledge and Belief. Ithaca, NY: Cornell University Press, 1962.

[Horgan and Tienson, 1988] T. Horgan and J. Tienson. Settling into a new paradigm. Southern Journal of Philosophy, 26:97-113, 1988. Connectionism and the Philosophy of Mind: Proceedings of the 1987 Spindel Conference, special supplement.

[Horgan and Tienson, 1989] T. Horgan and J. Tienson. Representations without rules. Philosophical Topics, 17:147-174, 1989.

[Horgan and Tienson, 1990] T. Horgan and J. Tienson. Soft laws. In Peter A. French, Theodore E. Uehling, Jr., and Howard K. Wettstein, editors, The Philosophy of the Human Sciences, volume 15 of Midwest Studies in Philosophy, pages 256-279. Notre Dame, IN: University of Notre Dame Press, 1990.

[Horgan and Tienson, 1992] T. Horgan and J. Tienson. Cognitive systems as dynamical systems. Topoi, 11:27-43, 1992.

[Horgan and Tienson, 1996] Terence Horgan and John Tienson. Connectionism and the Philosophy of Psychology. Cambridge, MA: MIT Press, 1996.

[Horgan and Tienson, 1999a] T. Horgan and J. Tienson. Authors' replies. Acta Analytica, 22:275-287, 1999.

[Horgan and Tienson, 1999b] T. Horgan and J. Tienson. Short précis of Connectionism and the Philosophy of Psychology. Acta Analytica, 22:9-21, 1999.

[Humphreys et al., 1989] M.S. Humphreys, J.D. Bain, and R. Pike. Different ways to cue a coherent memory system: A theory for episodic, semantic and procedural tasks. Psychological Review, 96:208-233, 1989.

[Hutchins, 1995] E. Hutchins. Cognition in the Wild. Cambridge, MA: MIT Press, 1995.

[Krifka et al., 1995] Manfred Krifka, Francis Jeffry Pelletier, Gregory N. Carlson, Alice ter Meulen, Godehard Link, and Germano Chierchia. Genericity: An introduction. In Gregory N. Carlson and Francis Jeffry Pelletier, editors, The Generic Book, pages 1-124. Chicago, IL: The Univeristy of Chicago Press, 1995.

[Landauer and Dumais, 1997] T.K. Landauer and S.T. Dumais. A solution to Plato's problem: The latent semantic analysis theory of acquisition, induction and representation of knowledge. Psychological Review, 104:211-240, 1997.

[Landauer et al., 1998] T.K. Landauer, P.W. Foltz, and D. Laham. An introduction to latent semantic analysis. Discourse Processes, 25(2\&3):259-284, 1998. 
[Levy and Bullinaria, 1999] J.P. Levy and J.A. Bullinaria. Learning lexical properties from word usage patterns: Which context words should be used? In R.F. French and J.P. Sounge, editors, Connectionist Models of Learning, development and Evolution: Proceedings of the Sixth Neural Computation and psychology Workshop, pages 273282. Springer, 1999.

[Lowe, 2000] W. Lowe. What is the dimensionality of human semantic space? In Proceedings of the 6th Neural Computation and Psychology workshop, pages 303-311. Springer Verlag, 2000.

[Lowe, 2001] W. Lowe. Towards a theory of semantic space. In J. D. Moore and K. Stenning, editors, Proceedings of the Twenty-Third Annual Conference of the Cognitive Science Society, pages 576-581. Lawrence Erlbaum Associates, 2001.

[Lund and Burgess, 1996] K. Lund and C. Burgess. Producing high-dimensional semantic spaces from lexical co-occurrence. Behavior Research Methods, Instruments 8 Computers, 28(2):203-208, 1996.

[Minsky, 1975] Marvin Minsky. Frame-system theory. In R.C. Schank and B.L. NashWebber, editors, Interdisciplinary Workshop on Theoretical Issues in Natural Language Processing. Cambridge, MA: M.I.T Press, 1975. Preprints of a conference at MIT, June 1975. Reprinted in P.N. Johnson-Laird and P.C. Wason, editors. Thinking: Readings in Cognitive Science, Cambridge: Cambridge University Press 1977; pp. 355-376.

[Norman, 1993] D.A. Norman. Things That Make Us Smart: Defending Human Attributes in the Age of the Machine. Reading, MA: Addison-Wesley, 1993.

[Patel et al., 1997] M. Patel, J.A. Bullinaria, and J.P. Levy. Extracting semantic representations from large text corpora. In R.F. French and J.P. Sounge, editors, Connectionist Models of Learning, Development and Evolution: Proceedings of the Fourth Neural Computation and Psychology Workshop, pages 199-212. Springer, 1997.

[Quine, 1960] W.V. Quine. Word and Object. Cambridge, MA and New York: MIT Press and John Wiley, 1960.

[Quine, 1969] W.V. Quine. Natural kinds. In Nicholas Rescher, editor, Essays in Honor of Carl G. Hempel, pages 5-23. Dordrecht: Reidel, 1969.

[Quine, 1970] W.V. Quine. Philosophy of Logic. Englewood Cliffs, NJ: Prentice-Hall, 1970.

[Rensink, 2000] R. Rensink. Visual sensing without seeing. Psychological Science, 15:27$32,2000$.

[Rescher, 1996] Nicholas Rescher. Priceless Knowledge? Natural Science in Economic Perspective. Lanham, MD: Rowman and Littlefield, 1996.

[Rosch, 1978] Eleanor Rosch. Principles of categorization. In Eleanor Rosch and B.B. Lloyd, editors, Cognition and Categorization, pages 27-48. Hillsdale, NJ: Erlbaum, 1978.

[Sahlgren, 2002] M. Sahlgren. Towards a Flexible Model of Word Meaning. Paper presented at the AAAI Spring Symposium 2002, March 25-27, Stanford University, Palo Alto, California, USA, 2002.

[Schank and Abelson, 1977] Roger Schank and Robert Abelson. Scripts, Plans, Goals and Understanding: An Inquiry into Human Knowledge Structures. Hillsdale, NJ: Lawrence Erlbaum Associates, 1977.

[Schütze, 1998] H. Schütze. Automatic word sense discrimination. Computational Linguistics, 24(1):97-124, 1998.

[Shannon, 1993] B. Shannon. The Representation and the Presentational: An Essay on Cognition and the Study of Mind. New York and London: Harvester Wheatsheaf, 1993.

[Shiffrin, 1997] Richard M. Shiffrin. Attention, automatism and consciousness. In Jonathan D. Cohen and Jonathan W. Schooler, editors, Scientific Approaches to Consciousness, pages 49-64. Mahwah, NJ: Erlbaum, 1997.

[Simon, 1957] H.A. Simon. Models of Man. New York: John Wiley, 1957. 
[Smith and Medin, 1981] Edward E. Smith and Douglas L. Medin. Categories and Concepts. Cambridge, MA: Harvard University Press, 1981.

[Stanovich, 1999] Keith A. Stanovich. Who is Rational? Studies of Individual Differences in Reasoning. Mahwah, NJ: Erlbaum, 1999.

[Sterelny, 1990] K. Sterelny. The Representation Theory of Mind. Oxford: Blackwell, 1990.

[Tallis, 1999] Raymond Tallis. The Explicit Animal: A Defence of Human Consciousness. London: Macmillan and New York: Martin's Press, 2nd edition, 1999.

[Tenenbaum et al., 2000] Joshua B. Tenenbaum, Vin de Silva, and John C. Langford. A global geometric framework for nonlinear dimensionality reduction. Science, 290(5500):2319-2323, December 2000.

[Thagard, 1989] Paul Thagard. Explanatory coherence. Behavioral and Brain Sciences, 12:381-433, 1989.

[Thelen and Smith, 1993] E. Thelen and I.B. Smith. A Dynamic Systems Approach to the Development of Cognition and Action. Cambridge, MA: MIT Press, 1993.

[van Rijsbergen, 2004] C.J. van Rijsbergen. The Geometry of Information Retrieval. Cambridge University Press, 2004.

[Webb, 1994] B. Webb. Robotic experiments in cricket phonotaxis. In D. Cliff, P. Husbands, J.A. Meyer, and S. Wilson, editors, From Animals to Animats 3: Proceedings of the Third International Conference on Simulation of Adaptive Behavior, pages 45-54. Cambridge, MA: MIT Press/Bradford Books, 1994.

[Wheeler, 1994] M. Wheeler. From activation to activity: Representation, computation and the dynamics of neural network control systems. Artificial Intelligence and Simulation of Behaviour Quarterly, 87:36-42, 1994.

[Wheeler, 2001] M. Wheeler. Two threats to representation. Synthese, 129:211-231, 2001.

[Widdows and Higgins, 2004] D. Widdows and M. Higgins. Geometric ordering of concepts, logical disjuntion, and learning by induction. In Compositional Connectionism in Cognitive Science, 2004. AAAI Fall Symposium Series.

[Widdows and Peters, 2003] D. Widdows and S. Peters. Word vectors and quantum logic: experiments with negation and disjunction. In Proceedings of Mathematics of Language 8, pages 141-154, 2003.

[Widdows, 2003] Dominic Widdows. Orthogonal negation in vector spaces for modelling word-meanings and document retrieval. In Proceedings of the 41st Annual Meeting of the Association for Computational Linguistics (ACL), Sapporo, Japan, 2003.

[Wiles et al., 1994] J. Wiles, G.S. Halford, J.E.M. Stewart, M.S. Humphreys, J.D. Bain, and W.H. Wilson. Tensor Models: A creative basis for memory and analogical mapping. In T. Dartnall, editor, Artificial Intelligence and Creativity, pages 145-159. Kluwer Academic Publishers, 1994.

[Wimsatt, 1986] W. Wimsatt. Forms of aggregativity. In A. Donagan, N. Perovich, and M. Wedin, editors, Human Nature and Natural Knowledge, pages 259-293. Dordrecht: Reidel, 1986.

[Wolfram, 1984] Stephen Wolfram. Computer softwear in science and mathematics. Scientific American, 251:188, September 1984.

[Woods, 2004] John Woods. The Death of Argument: Fallacies in Agent-Based Reasoning. Dordrecht and Boston: Kluwer, 2004.

[Zimmermann, 1989] Manfred Zimmermann. The nervous system and the context of information theory. In R.F. Schmidt and G. Thews, editors, Human Physiology, pages 166-175. Berlin: Springer-Verlag, 2nd edition, 1989. Marguerite A. BiedermanThorson, translator.

[Zipf, 1949] George K. Zipf. Human Behavior and the Principle of Least Effort. Cambridge, MA: Addison Wesley, 1949. 